\title{
Use of murine models to identify tumor immune components that correlate with response to immunotherapy in breast cancer
}

Jessica A. Castrillon ${ }^{1,2}$, Anita K. Mehta ${ }^{1,2}$, Emily Cheney ${ }^{1}$, Eric Miller ${ }^{3}$, Alaba Sotayo ${ }^{2}$, Elizabeth A. Mittendorf ${ }^{1,4,5}$, Anthony Letai ${ }^{2,6}$, and Jennifer L. Guerriero ${ }^{1,2,5,6,7}$

${ }^{1}$ Breast Tumor Immunology Laboratory, Susan F. Smith Center for Women's Cancers, DanaFarber Cancer Institute, Boston, MA

2Department of Medical Oncology, Dana-Farber Cancer Institute, 450 Brookline Avenue, Boston, MA 02215, USA

${ }^{3}$ Nanostring Technologies, Seattle, WA, USA

${ }^{4}$ Division of Breast Surgery, Department of Surgery, Brigham and Women's Hospital, Boston, MA ${ }^{5}$ Breast Oncology Program, Dana-Farber/Brigham and Women's Cancer Center, Boston, MA ${ }^{6}$ Ludwig Center for Cancer Research at Harvard, Harvard Medical School, Boston, MA

${ }^{7}$ Corresponding author

Corresponding author: Jennifer L. Guerriero, Dana-Farber Cancer Institute, 450 Brookline Avenue, Boston, MA, 02215. Jennifer_Guerriero@dfci.harvard.edu, 617-582-9759.

Running title: (60 characters): Murine models for breast cancer immunotherapy 


\section{Abstract}

Background: The heterogeneity of the breast tumor microenvironment (TME) may contribute to the lack of durable responses to immune checkpoint blockade (ICB), however, mouse models to test this are currently lacking. Proper choice and use of pre-clinical models are necessary for rigorous, pre-clinical studies to rapidly move laboratory findings into the clinic to treat patients.

Methods: To elucidate how tumor latency and the heterogeneity of the TME contributes to ICB resistance, we performed comprehensive characterization of the TME using quantitative flowcytometry and RNA expression analysis (NanoString) utilizing three distinct breast cancer models, all derived from the same autochthonous model. Tumor cells were obtained from the commonly used MMTV-PyMT murine breast cancer model and 1E6, 1E5 or 1E4 cells were either immediately injected into the mammary fat pad of FVB/NJ wild type mice or frozen (i.e. the tumor inoculation derived from the MMTV-PyMT tumors were never cultured). We then correlated the immunophenotyping to the efficacy observed from ICB.

Results: These studies reveal that the number of cells used to generate syngeneic tumors significantly influences tumor latency, the infiltrating leukocyte population and response to ICB. The 4 models had vastly different TMEs which correlated to responses to ICB. Compared to the autochthonous model, all three syngeneic models had significantly more tumor infiltrating lymphocytes (TILs; $\mathrm{CD}^{+}, \mathrm{CD}^{+}$, and $\mathrm{CD} 8^{+}$) and higher proportions of PD-L1 positive myeloid cells, whereas the MMTV-PyMT model had the highest frequency of myeloid cells out of total leukocytes. Increased TILs correlated with response to anti-PD-L1 and anti-CTLA-4 therapy; but tumor cell PD-L1 expression and T-cell PD-1 expression did not.

Conclusions: Here we have identified ICB-sensitive and resistant breast cancer models, generated from the same tumor cell inoculum. These models represent an opportunity to further interrogate the TME for breast cancer treatment and provide novel insights into therapeutic combinations and response to ICB. We believe this work serves as an important resource for the field to inform proper mouse model selection for pre-clinical studies.

Keywords: breast cancer, immunotherapy, immune checkpoint blockade, syngeneic tumor models, preclinical mouse models 


\section{Background}

The success of immune checkpoint blockade (ICB) in a variety of human cancer types has stimulated interest in its use for the treatment of breast cancer. Current therapy for breast cancer is guided by the molecular pathology of the tumor. Breast cancer is often driven by overactive hormone signaling (estrogen and/or progesterone receptors; ER, PR) or amplification of growth factor response (HER2) and therefore treated with endocrine therapy or HER2-targeted agents; alternatively patients can also be treated with general therapies such as chemotherapy and/or radiation. ${ }^{1-4}$ After initial treatment for early stage disease, approximately $30 \%$ of women will eventually develop recurrent advanced or metastatic disease. ${ }^{5}$ Almost all who develop metastatic breast cancer will succumb to the disease, highlighting the need for more effective strategies. ${ }^{6}$ ICB aims to target T-cell inhibitory molecules using antibodies against cytotoxic T-lymphocyte associated protein 4 (CTLA-4) and programmed cell death protein-1 (PD-1) as well as its ligand (PD-L1). ICB works by reinvigorating anti-tumor immune responses by inhibiting negative interactions between T-cells and antigen presenting cells (APCs) or tumor cells in several cancer types. ${ }^{7,8}$ In 2011, the first ICB agent, ipilimumab, a human monoclonal antibody targeting CTLA4, was approved by the FDA for treatment of metastatic melanoma based on significant improvement in overall survival in a randomized, double-blinded Phase III study. ${ }^{9}$ Importantly, ipilimumab has doubled 10-year survival for metastatic melanoma compared with historical data. ${ }^{10-12}$ Antibodies targeting PD-1 and PD-L1 have also shown a durable clinical response in melanoma, as well as renal cell carcinoma, non-small cell lung cancer, and bladder cancer. ${ }^{13-20}$ To date, such responses have led to FDA approval of seven ICB therapies for the treatment of more than 15 different types of cancer. ${ }^{21}$ Because their effector pathways are distinct, there are reasons to believe that the combination of CTLA-4 and PD-1/PD-L1 therapy can provide an enhanced response. ${ }^{22,23}$

While ICB has shown promising results in a subset of patients with other cancer types, responses in breast cancer has demonstrated only minimal responses..$^{24}$ Breast cancer has a lower mutational burden compared to other types of cancer which may explain the lack of efficacy in response to ICB. ${ }^{25,26}$ Despite this generalization, triple negative breast cancer (TNBC) has demonstrated some benefit from ICB therapy, albeit not achieving the response rates demonstrated in melanoma and lung cancer. While pembrolizumab (anti-PD-1) showed promising activity as a single agent against advanced or metastatic TNBC in the KEYNOTE-012 (NCT01848834) and KEYNOTE-086 (NCT02447003) clinical trials, in the randomized, Phase III KEYNOTE-119 (NCT02555657) clinical trial there was no improvement in overall survival 
compared to single-agent chemotherapy in metastatic TNBC. ${ }^{27}$ Benefit from ICB therapy has been observed in patients treated in the first line setting and/or in patients whose tumors or immune cells express PD-L1. ${ }^{28-30}$ For example, in the PCD4989g (NCT01375842) clinical trial, evaluating Atezolizumab as a single agent, expression of PD-L1 on $1 \%$ or greater of immune infiltrating cells was associated with a $12 \%$ ORR compared to $0 \%$ when there was no expression of PD-L1, and high levels of immune cell infiltration (greater than 10\%) was independently associated with higher overall response rate (ORR) and overall survival (OS). ${ }^{29}$ Importantly, the first FDA approval of ICB in breast cancer came from the Phase III IMpassion130 (NCT02425891) clinical trial that demonstrated atezolizumab in combination with nab-paclitaxel showed significant extension of median disease-free overall survival compared to nab-paclitaxel alone, from 15.5 to 25 months in patients with $1 \%$ or more PD-L1 positive immune cells, where there was no benefit in PD-L1 negative tumors. ${ }^{31,32}$

The use of PD-L1 as a biomarker for ICB has been rigorously investigated but has raised concerns including poor agreement between different antibodies as well as scoring between pathologists. ${ }^{33}$ To date, there are 9 FDA approvals for the use of ICB based on a specific PD-L1 threshold and companion diagnostic, with variable thresholds both within and across tumor types using several different assays, including approvals at the following PD-L1 positive percentage thresholds: 1,5 , and $50 \%$. In a recent meta-analysis that examined all approvals of ICB as of April 2019, PD-L1 was predictive in $28.9 \%$ of those approvals, and was either not predictive (53.3\%) or not tested $(17.8 \%)$ in the remaining approvals. ${ }^{21}$ Other predictors of response to ICB include the presence of immune cells, as immune cells within HER2 ${ }^{+}$and TNBC tumors have been shown to correlate with better response to HER2-targeted therapy and chemotherapy, respectively. ${ }^{34}$ However, immune cell infiltration has been reported to differ among each subtype of breast cancer. ${ }^{35}$ Further work to characterize the TME of breast tumors will provide opportunity for ICB therapy in these patients.

Mouse models have been instrumental in understanding the molecular mechanisms of oncogenesis and metastasis. Being able to translate in vivo pre-clinical findings to patients depends largely on how accurately the mouse model replicates histological markers, biochemical pathways, and genetic aberrations observed in the same human tumor type. ${ }^{36}$ In light of the advances in immunotherapy, it is now also necessary to meticulously characterize the TME of pre-clinical mouse models. Therefore, here we have generated and characterized three models of breast cancer, derived from a commonly used pre-clinical autochthonous model of breast cancer, in which the polyoma middle T (PyMT) oncogene is driven by the mouse mammary tumor 
virus (MMTV)-LTR. The MMTV-PyMT model is representative of human breast carcinomas; where several of the same signaling transduction pathways that are commonly disrupted in human breast cancer patients are seen in the MMTV-PyMT model such as Src family, Ras and PI3K kinase pathways. ${ }^{37,38}$ In addition, both innate and adaptive immune cells infiltrate into the tumor during tumorigenesis. ${ }^{39,40}$ Macrophages have been shown to play a key role in the development of these tumors, in which CCL2 recruits inflammatory monocytes to facilitate breast tumor growth and metastasis. ${ }^{41}$ Additionally, it's been shown that the phenotype is mediated through IL-4 expressing CD4 ${ }^{+} \mathrm{T}$-cells. ${ }^{40} \mathrm{~A}$ spectrum of macrophage phenotypes have been recognized, ranging from classically activated macrophages ("M1"-like) that are effective in clearing intracellular pathogens and can recruit cytotoxic $T$ lymphocytes to activate adaptive immune responses 42 ; to alternatively activated macrophages ("M2"-like), which function to help with parasite clearance, exhibit tissue remodeling capabilities and promote tumor progression by recruiting T regulatory and Th2 T-cell subsets lacking cytotoxic functions. ${ }^{43}$ Tumor associated macrophages (TAMs) likely exhibit features of both M1- and M2-like macrophages but in general, exhibit an M2-like phenotype and promote tumor progression and metastasis by secreting factors that regulate angiogenesis and that recruit tumor suppressive cells such as T regulatory (Treg) cells. ${ }^{44,45}$ In line with the lack of clinical efficacy of ICB in breast cancer, several studies have shown that MMTV-PyMT mice are resistant to ICB monotherapy. $39,46,47$

With respect to ICB therapy in the clinical care of breast cancer patients, it is currently unclear if immune cell infiltration, including type, number and/or phenotype, correlates to responses. Understanding what factors are critical for ICB efficacy in breast cancer will allow careful patient selection and/or catalyze clinical development of novel therapies to convert nonresponders to responders, deepen responses that do occur, surmount acquired resistance to immunotherapy and identify biomarkers that can more accurately predict durable response. Therefore, here we generate three distinct syngeneic models derived from the MMTV-PyMT model and provide deep immuno-phenotypic analysis of biomarkers and mechanisms that correlate with efficacy to ICB therapy. 


\section{Methods}

\section{Animal husbandry}

All experiments used either virgin female $\mathrm{FVB} / \mathrm{NJ}$ mice or virgin female $\mathrm{FVB} / \mathrm{N}$ autochthonous mice carrying the polyoma middle T (PyMT) transgene under the control of the mammary tumor virus (MMTV) promoter were used. The FVB/NJ mice were purchased from Jackson laboratory (001800). All mice were maintained within the Dana-Farer Cancer Center (DFCl) and all experiments were conducted under The Institutional Animal Care and Use Committee (IACUC).

\section{Generation of syngeneic models}

Late stage MMTV-PyMT tumors were harvested and tumor suspension was either immediately injected into recipient FVB/NJ wild-type mice or frozen for subsequent experiments. The tumor suspension was never cultured. Each experiment was performed with 3 different batches of cells harvested from MMTV-PyMT mice. FVBN/J mice were inoculated with one million (1E6), one hundred thousand (1E5), or ten thousand (1E4) cells in the $4^{\text {th }}$ mammary fat pad to generate syngeneic models.

\section{Tumor digestion}

Tumors were extracted and minced, and subsequently blended using the gentleMACS Dissociator (Miltenyi Biotec cat. \#130-093-235). MACS Miltenyi Tumor Dissociation Kit for mouse (Miltenyi Biotec cat. \#130-096-730) was used for further enzymatic digestion according to the manufacturer's protocol. Dissociated tumor cell suspensions were rinsed with RPMI Medium 1640 (Life Technologies cat. \# 11875-093), filtered using a $70 \mu \mathrm{m}$ sterile EASY-strainer ${ }^{\mathrm{TM}}$ (Greiner bioone cat. \#542 070) and performed red blood cell lysis using RBC Lysis Solution (Qiagen cat. \#158904).

\section{Efficacy studies}


Caliper measurements were used to calculate tumor volumes for each mammary tumor using [(Length $\times$ Width $\left.{ }^{2} / 2\right]$. MMTV-PyMT mice were enrolled into as study at about 80 days old and when each tumor reached $80-100 \mathrm{~mm}^{3}$. Tumors from mammary fat pad numbers 5 and 10 were excluded from the analysis. The sum of the volumes for the MMTV-PyMT autochthonous tumors (1-4 and 5-9) were used and indicated as "total tumor burden". The syngeneic mice that had tumors that measured $80-100 \mathrm{~mm}^{3}$ were enrolled into an experiment. At the indicated time points animals were euthanized in a $\mathrm{CO}_{2}$ chamber before performing a cardiac perfusion with normal saline. Lungs and tumors were removed for analysis.

\section{Flow cytometry}

Tumors were digested as described above, and single cells were re-suspended in a buffer containing 2\% FBS and 2mM EDTA (Sigma-Aldrich cat. \#E7889) diluted in Phosphate buffered saline (PBS) (Lift Technologies cat. \#10010-023). Zombie Aqua ${ }^{\mathrm{TM}}$ Fixable Viability Kit (BioLegend cat. \#423101) and anti-mouse CD16/CD32 Fc gamma receptor II/III blocking antibody (Affymetrix cat. \#14-0161) was diluted in PBS and applied to cells for 20 minutes in the dark on ice. Cells

were washed and incubated with fluorochrome-conjugated antibodies (anti-mouse CD45 Alexa Fluor ${ }^{\circledR}$ 488, clone 30-F11, BioLegend cat. \#103122; anti-mouse CD11b Brilliant Violet 711, clone M1/70, BioLegend cat. \#101241; anti-mouse CD3 Alexa Fluor® 594, clone 17A2, BioLegend cat. \#100240; anti-mouse MHCII Brilliant Violet 421, clone M5/114.15.2, BioLegend cat. \#107631; anti-mouse F4/80 Alexa Fluor® 647, clone BM8, BioLegend cat. \#123122; anti-mouse CD11c Brilliant Violet 650, clone HL3, BD Biosciences cat. 564079; anti-mouse CD80 Brilliant Violet 605, clone 16-10A1, BioLegend cat. \#104729; anti-mouse CD86 PerCP/Cy5.5, clone GL-1, BioLegend cat. \#105027; anti-mouse CD40 PE/Cy7, clone 3/23, BioLegend cat. \#124621; anti-mouse CD206 PE, clone C068C2, BioLegend cat. \#141706; anti-mouse CD8 PE/Cy7, clone 53-6.7, BioLegend cat. \#100721; anti-mouse CD4 PE, clone GK1.5, BioLegend cat. \#100408; anti-mouse Ly-6G/Ly6C (GR1) Brilliant Violet 650, clone RB6-8C5, BioLegend cat. \#108441; anti-mouse PD-1 Brilliant 
Violet 421, clone 29F.1A12, BioLegend cat. \#135217; anti-mouse PDL-1 PE, clone 10F.9G2, BioLegend cat. \#124307) in the dark for an hour according at the dilution recommended by the manufacturer. Following staining, cells were rinsed with PBS buffer and fixed with $1 \%$ paraformaldehyde for 5 minutes at room temperature. Afterwards, cells were rinsed with PBS, resuspended in PBS buffer, and placed in the dark at $4^{\circ} \mathrm{C}$ until analysis. Following extracellular staining, cells that obtained an intracellular stain were washed, fixed, and permeabilized using the Foxp3/ Transcription Factor Staining Buffer Set Kit (Affymetrix cat. \#00-5523) according to the manufacturer's protocol. Cells were incubated with antibody (anti-mouse Granzyme B Alexa Fluor® 647, clone GB11, BioLegend cat. \#515405; anti-mouse FoxP3 PerCP/C5.5, clone R16$715 \mathrm{BD}$, Biosciences cat. \#563902) overnight in the dark at $4^{\circ} \mathrm{C}$. The following day, cells were rinsed with PBS, and re-suspended with PBS buffer for flow cytometric analysis on the BD LSRFortessa at the Hematologic Neoplasia Flow Cytometry Core of the Dana-Farber Cancer Institute. Five hundred thousand to two million cells were analyzed per sample per mouse using BD FACs Diva Software. Single color controls were included to QC analysis. Data analysis and compensation were performed on BD FACS Diva software. The absolute cell number populations was calculated using the equation below:

$$
\text { absolute cell population }=\frac{\% \text { cell population } \text { viable }^{*} \mid \text { viable cells } \mid}{100 * \text { tumor }_{\text {weight }}}
$$

\section{Dosing}

All in vivo experiments were treated with intraperitoneal injections. Mice were treated twice a week with 200 ug of InVivoMAb rat IgG2b isotype control, anti-keyhole limpet hemocyanin (clone LTF-2, BioXcell BE0090), InVivoMab anti-mouse CTLA-4 (clone 9H10, BioXcell BE 0131), and InVivoMab anti-mouse PDL-1 (clone 10F9G2, BioXcell BE0101). Mice were treated until tumors reached $2 \mathrm{~cm}$ in one direction.

\section{RNA isolation}


When syngeneic mouse tumors reached $100 \mathrm{~mm}^{3}$, tissue samples were snap frozen for later processing. Samples were also collected from autochthonous mice with total tumor burden in the range of $300-600 \mathrm{~mm}^{3}$. Tumor specimens of $30 \mathrm{mg}$ were used for RNA isolation using the RNeasy Mini Kit (Qiagen cat. \#74104). $\beta$-Mercaptoethanol was added to Buffer RLT and subsequently added to each tumor sample. Tissue was disrupted and homogenized using a 20-gauge needle. An equal volume of $70 \%$ ethanol was added, transferred to a RNeasy spin column and centrifuged for 30 seconds at 12,000 rpm. The flow-through was discarded. Buffer RW1 was added to the RNeasy spin column and centrifuged at $12,000 \mathrm{rpm}$ for 30 seconds. The flow-through was discarded. Residual DNA was removed using the RNase-Free DNase Set (Qiagen cat. \#79254) according to the manufacturer's protocol. RPE was added to the RNeasy spin column and centrifuged at 12,000 rpm for 30 seconds. This step was repeated and centrifuged at the same speed for 2 minutes. RNeasy spin column was placed in a clean $2 \mathrm{ml}$ collection tube. Samples were eluted with 50 ul of RNase-free water for 1 minute at 12,000 rpm. Samples were analyzed by the nanodrop to detect concentration and 260/230 ratio. RNA purity was assessed using the Agilent Bioanalyzer 4200 at the Molecular Biology Core Facility of Dana-Farber Cancer Institute.

\section{Immune profile gene analysis}

Purified RNA was isolated from samples from mice when individual tumors reached 100 $\mathrm{mm}^{3}$. Isolated RNA was submitted to the Center for Advanced Molecular Diagnostics core facility at Brigham and Women's Hospital. Gene expression analysis was conducted using the nCounter PanCancer Immune Profiling panel which includes 770 immune-related genes and relevant controls. NanoString gene expression values were normalized using the best subset of the 40 reference genes included in the panel, as determined by the geNorm algorithm ${ }^{48}$. The nSolver Advanced Analysis 2.0 software was used to perform all normalization. Pathway signatures were calculated by condensing biologically related groups of genes using the first principal component of their expression data. ${ }^{49}$ Cell type scores were calculated using the average log2 normalized 
expression of each cell type's marker genes. The cell type abundance scoring is modified from other reports ${ }^{50}$ where strict cell type gene correlation-driven QC $p$-values were determined based on data that passed QC. The cell scoring employed within the manuscript makes assumptions that result in the cell typing data being best described as trends. 


\section{Results}

\section{Tumor infiltrating leukocyte populations are significantly altered in the 4 pre-clinical murine breast cancer models.}

Three distinct pre-clinical models of breast cancer were generated by isolating tumor cells (both tumor and immune) from autochthonous MMTV-PyMT tumors and directly (i.e. never culturing the suspension) inoculating $1 \mathrm{E} 6,1 \mathrm{E} 5$ or $1 \mathrm{E} 4$ cells, into the $4^{\text {th }}$ mammary fat pad of wildtype FVB/NJ mice (Figure 1a). Flow cytometry was used to assess the composition of cells that were injected into recipient mice (gating strategy in Supplemental Figure 1a), which revealed that the cell suspension was approximately $85 \%$ tumor cells (CD45) and $15 \%$ immune cells (CD45+; Supplemental Figure $1 \mathrm{~b}-\mathrm{c})$. Tumor latency was directly related to the number of cells that were inoculated into the mammary fat pad, as inoculating fewer cells correlated with a longer time to reach $100 \mathrm{~mm}^{3}$ (Figure 1b). It took approximately 10, 15, and 30 days for tumors to reach a volume of $80-100 \mathrm{~mm}^{3}$ in the $1 \mathrm{E} 6,1 \mathrm{E} 5$, and 1E4 models, respectively, while it took approximately 80 days for each tumor on the autochthonous model to reach $100 \mathrm{~mm}^{3}$ (Figure $1 \mathrm{~b}$ ).

To test if tumor latency influences immune cell infiltration or phenotype, immunophenotyping was performed on each of the breast cancer models. Tumors were harvested when they reached $100 \mathrm{~mm}^{3}$ for baseline immune profiling because this is generally the size of tumors when animals are enrolled into pre-clinical efficacy studies. Flow cytometry revealed significant difference in the frequency of leukocytes (CD45+ cells) within the tumor as a percentage of total viable cells (Figure 1c). Tumors with the shortest latency (1E6 model) had the highest absolute number of infiltrating leukocytes as measured by CD45+ cells out of total viable cells (Figure 1c). Strikingly, in the 1E6 and 1E5 models, immune cells were the major cell type within tumors, representing more than $60 \%$ of the total cells in the tumor which may represent an acute inflammatory response from the inoculation. T-cells (percent of $\mathrm{CD}^{+}$cells out of total of viable cells) accounted for a substantial fraction of the CD45 cells in the syngeneic models and was less robust in the 1E4 and transgenic models (Figure 1d,e). Myeloid cell infiltration (percent of CD11 $\mathrm{b}^{+}$cells out of total of viable cells) remained more consistent in their frequency, however the autochthonous model had significantly fewer myeloid cells than the 1E6 and 1E5 models (Figure 1d,e). This resulted in differences in the ratio of T-cells to myeloid cells, where tumor models with a shorter tumor latency had the highest ratios. The $1 \mathrm{E} 6$ model had $5.63 \mathrm{~T}$-cells to every 1 myeloid cell whereas the autochthonous model had 2.01 T-cells for every 1 myeloid cell (Figure 1f). Additionally, a correlation was observed between tumor latency and the number of Tcells within the tumor (Figure 1g). 
While significant differences in the numbers of T-cells and myeloid cells out of total viable cells in the tumor was identified (Figure 1d), the total number of $\mathrm{CD} 45^{+}$immune cells was significantly different between the models (Figure 1c). Therefore, further analysis was performed to determine the proportion of T-cells and myeloid cells out of gram of tumor tissue as well as their frequency out of $\mathrm{CD} 45^{+}$cells. The extent of T-cell infiltration out of gram of tissue (Figure 2a) was similar to the findings out of total viable cells (Figure 1c), which reflects the absolute increase in numbers of T-cells out of all cells in the tumor. This trend was consistent with the absolute numbers of $\mathrm{CD}^{+}$and $\mathrm{CD} 4^{+} \mathrm{T}$-cells (Figure 2a). The same pattern emerged when gated as a frequency out of $\mathrm{CD} 45^{+}$cells (Figure $2 b$ ). Conversely, while the autochthonous model had the fewest number of myeloid cells out of viable cells (Figure 1d), as well as out of gram of tumor (Figure 2c), it had the highest frequency of myeloid cells and macrophages out of total CD45+ immune cells (Figure 2d). The other myeloid cell populations $\left(C D 11 c^{+}\right.$and $\left.G r 1^{+} C D 11 b^{+}\right)$were relatively low and unchanged between the distinct models, except for a small, yet significant increase in MDSCs $\left(\mathrm{Gr} 1^{+} \mathrm{CD} 11 \mathrm{~b}^{+}\right)$in the $1 \mathrm{E} 6$ compared to the $1 \mathrm{E} 4$ model (Figure $2 \mathrm{~d}$ ). There was a 1:1 ratio of $C D 11 b^{+}$to $C D 3^{+}$of $C D 45^{+}$cells in the transgenic model, which was the highest ratio among the four models (Fig. 2e). Therefore, while there are relatively few immune cells in the TME of MMTV PyMT tumors, there is a relatively high number of immune cells from the myeloid lineage.

\section{Subset characterization reveals differences in tumor T-cell immune populations among the 4 distinct breast cancer models.}

The difference in absolute numbers of T-cells and myeloid cells prompted further investigation into immune cell subpopulations and their respective phenotypes. Using flow cytometry, T-cell populations were characterized out of $\mathrm{CD}^{+}$cells including $\mathrm{CD}^{+} \mathrm{FoxP}^{+}(\mathrm{T}-$ regulatory cells; Tregs) as well as $\mathrm{CD} 8^{+} \mathrm{T}$-cells. The Treg population increased in the $1 \mathrm{E} 5$ and $1 E 4$ models (Figure $3 a, b$ ) and there was no change in the frequency of CD8 T-cells out of total $\mathrm{CD}^{+}$cells (Figure $3 \mathrm{c}-\mathrm{e}$ ). The ratio of $\mathrm{CD} 4^{+} \mathrm{CD} 3^{+}$cells over $\mathrm{CD} 8^{+} \mathrm{CD} 3^{+}$cells was highest in the $1 \mathrm{E} 6$ model (Figure 3f). A different gating strategy was used to identify the frequency of FoxP3 ${ }^{+}$ cells out of $\mathrm{CD}^{+}{ }^{+} \mathrm{T}$-cells (Tregs) as well as the frequency of granzyme $\mathrm{b}(\mathrm{GrB})^{+} \mathrm{CD} 8^{+} \mathrm{T}$-cells (cytotoxic T lymphocytes; CTLs). This revealed that $1 \mathrm{E} 5$ and $1 \mathrm{E} 6$ models again had the highest frequency of Tregs whereas the autochthonous model had the highest frequency of $\mathrm{GrB}^{+} \mathrm{CD} 8^{+}$ CTLs (Figure $3 g, h$ ). This resulted in a significant increase in the CTL:Treg ratio in the autochthonous model compared to the 1E5 and 1E4 model (Figure 3i). 


\section{Subset characterization reveals differences in tumor myeloid immune cell populations among the breast cancer models.}

Further analysis of the $\mathrm{CD} 11 \mathrm{~b}^{+}$cells revealed significant differences of myeloid subpopulations between the four distinct tumor models (Figure 4a-e). The autochthonous and 1E4 models had the highest proportion of $\mathrm{Gr}^{+}{ }^{+}$cells out of $\mathrm{CD}_{11} \mathrm{~b}^{+}$cells; this phenotype is generally characterized as myeloid derived suppressor cells ${ }^{51,52}$ (Figure 4a). The F480 ${ }^{+}$cells in the autochthonous model were largely PD-L1 negative, in contrast to $\mathrm{F} 480^{+}$cells in the syngeneic models, which had a higher proportion of PD-L1 positive cells (Figure 4b,c,e). A different gating strategy was used to assess PD-L1+ cells out of $\mathrm{CD} 11 \mathrm{~b}^{+}$as well as of CD45- (tumor cells). The frequency of PD-L1 expression on myeloid cells was lowest in the autochthonous model, and there was no change on tumor cells (Figure $4 \mathrm{f}, \mathrm{g}$ ), and that the autochthonous model had the highest frequency of PD-1+ T-cells out of $\mathrm{CD} 3^{+} \mathrm{CD} 45^{+}$cells (Figure $4 \mathrm{~h}$ ).

Our group previously described that converting pro-tumor macrophages to an anti-tumor phenotype induced reduction of primary and metastatic tumors, indicating that the myeloid cell population is a major contributor to disease progression. ${ }^{39}$ The phenotype of tumor macrophages has previously been shown to correspond to drug sensitivity and disease outcome in this model, therefore we further investigated macrophage phenotype across the different models ${ }^{39,40}$. Markers CD40, CD80 and CD86 were used to identify "M1"-like macrophages and CD206 was used as a marker "M2"-like macrophages. The autochthonous model and the 1E4 model had the highest frequency of M1-like myeloid and macrophage populations (Figure 4i and Supl. Fig. 3a-c) suggesting that the $1 \mathrm{E} 6$ and 1E5 models had more suppressive myeloid cells, in line with their higher frequencies of PD-L1+ myeloid cells.

\section{The breast cancer models have distinct tumor immune transcriptional profiles.}

To interrogate mRNA transcripts expressed by cells in each of the distinct tumors, transcriptional profiling was performed by NanoString analysis (Supplemental Figure 4). Since the analysis was performed on bulk tumor tissue, the data would correspond most similarly to flow cytometry analysis out of total viable cells and per gram of tumor tissue. The autochthonous tumors clustered most closely with the 1E4 tumors, and the 1E6 and 1E5 tumors clustered together (Supplemental Figure 4c,d). Cell type score analysis validated the flow cytometry analysis, where the $1 \mathrm{E} 6$ model had the highest scores for all immune cell types profiled, including T-cells and macrophages (Figure 5a-c and Supplemental Figure 4e). In addition, there was no change in neutrophils but the 1E6 and 1E5 model had the highest scores for dendritic cells, and 
the 1 E6 model had the highest score for activated natural killer cells (NK CD56dim; Supplemental Figure 4f).

Unsupervised hierarchical clustering was performed on activated pathway scores, and again, the 1E6 and 1E5 models clustered most similarly and the 1E4 and autochthonous models clustered most similarly (Figure 5c). Pathway analysis further revealed increased scores for immune related pathways including both adaptive and innate immunity, as well as macrophage and T-cell function. In general, the 1E6 model and 1E5 models had the highest scores for all immune related pathways (Figure $5 d$ and Supplemental Figure 5), likely due to the increased number of immune cells in the tumors that were identified by flow cytometry. The most striking differences were observed when comparing the autochthonous model with the 1E6 model, where multiple transcripts associated with antigen presentation and immune signaling were increased in the $1 \mathrm{E} 6$ model (Supplemental Figure 6a-f). More specifically, the $1 \mathrm{E} 6$ model had the highest levels of Cd274 (PD-L1), Ctla4, Ccr2 and Ccl2 (Figure 5e).

\section{The distinct models of MMTV-PyMT breast cancer respond differently to immune checkpoint blockade.}

We and others have previously shown that the MMTV-PyMT murine model of breast cancer is resistant to checkpoint blockade as a monotherapy. ${ }^{39,53}$ Here, the MMTV-PyMT model had the least number of infiltrating leukocytes and lymphocytes within the TME compared to the 3 syngeneic models. Since the syngeneic models had a higher absolute number of T-cells, as well as a higher proportion of T-cells expressing PD-1 and myeloid cells that express PD-L1, we hypothesized that the syngeneic models would demonstrate enhanced responses to ICB. Indeed, the 1E6 model, which had the highest frequency of PD-L1+ myeloid cells, was the only model among the four that responded to anti-PD-L1 and anti-CTLA-4 as monotherapy after 14 days of treatment as seen by delayed tumor progression (Figure 6, Supplemental Figure 7). This resulted in a small yet significant increase in overall survival (Fig. 6a). The combination of anti-PD-L1 plus anti-CTLA-4 was significant over vehicle control but not over either monotherapy. The 1 E5 model had a small yet significant decrease in total tumor burden with the anti-CTLA-4 monotherapy at day 14 , but did not translate to an increase in overall survival; however the combination of antiCTLA-4 plus anti-PD-L1 induced a reduction of tumor burden at 14 days that did lead to an improved overall survival (Figure 6b, Supplemental Figure 7b). Both the $1 \mathrm{E} 4$ and the autochthonous model were resistant to monotherapy as well as combination therapy (Figure 6cd, Supplemental Figure $7 \mathrm{c}-\mathrm{d}$ ). This data suggests that total immune cell infiltrate as well 
exhaustive immune cell phenotypes may correspond to the success of ICB therapy within the context of these breast cancer models (Figure 7).

To gain insight into what may correlate with ICB response, tumors were regrouped into either predicted responders (1E6 and 1E5 tumors) or predicted non-responders (1E4 and autochthonous; Supplemental Figure 8a). Predicted responders and non-responders clustered by immune cell score, where predicted responders had higher scores related to CD45, T-cells, cytotoxic T-cells, NK CD56 dim cells, macrophages and DCs (Supplemental Figure 8b,c). Responders and non-responders also clustered in the pathway analysis, where the inflammation pathway revealed significant differences between the responders and non-responders (Supplemental Figures 8d,e). 


\section{Discussion}

There is a critical need for biomarkers to predict response to ICB in breast cancer, and mouse models are currently lacking. Given the substantial heterogeneity of the TME, conclusions based on specific mouse models might limit generalizations, especially regarding detailed characterization of molecular signaling mechanisms. Here we exploited a syngeneic mouse model to make two major findings. First, the initiating conditions of the tumor (in this case, the number of cells in the inoculum) can dramatically alter the tumor immune microenvironment. Second, we found that these differences in the TME were closely related to quality of response to ICB. In this study we used cells derived from tumors that spontaneously arise in the MMTVPyMT murine model of breast cancer to generate three distinct syngeneic models using 1E6, 1 E5 or 1E4 cells injected into the mammary fat pad of wild-type FVB/NJ mice. Our findings are the first to report a detailed characterization of difference in the TME as a variable of the number of cells injected to generate syngeneic tumors. Importantly, we find that only the 1E6 and 1E5 models responded to ICB, whereas the 1E4 and MMTV-PyMT model are resistant. Our data suggest that protection from the inhibitory effects of Tregs and presence of high numbers of $\mathrm{T}$ cells and macrophages paired with enhanced antigen processing capabilities, as seen in the syngeneic models sensitive to anti-CTLA4 and anti-PD-L1 therapy, might be a setting where ICB may have great therapeutic potential for breast cancer. We found that the 1E6 model had the highest absolute number of T-cells and myeloid cells, and was responsive to ICB. Additionally, we found when comparing ICB-sensitive models (1E6 and 1E5) to the ICB-resistant models (1E4 and autochthonous), there is a significant difference in gene signatures related to antigen presentation and innate immune cell response. These data support our hypothesis that in addition to T-cells, macrophages and other myeloid cells are required to play a critical role in initiating an anti-tumor immune response.

Macrophages play an essential role in T-cell activation by presenting antigen and providing activating and stimulatory cytokines essential for T-cell function. ${ }^{54}$ In addition, macrophages can mediate antibody dependent cellular toxicity of cancer cells ${ }^{55}$ as well as eliminate cancer cells through Fc $\gamma$ R-mediated phagocytosis. ${ }^{56}$ However, TAMs can also dampen effector T-cell function by producing IL-10 that in turn increase their own PD-L1 expression and suppresses cytotoxic T-cell responses. ${ }^{57}$ Interestingly, we found that the ICB-sensitive models had the highest absolute number of $\mathrm{CD}_{11} \mathrm{~b}^{+}$myeloid and $\mathrm{F} 40^{+}$macrophages. These myeloid cells were more suppressive than those found in the ICB-resistant tumor models; indicated by a higher proportion of myeloid cells expressing PD-L1+ (Fig. 4f), as well as a lower ratio of M1:M2 
macrophages that suggested more M2-like macrophages (Supl. Fig. 3). In line with these observations, we found that transcripts levels related to $\mathrm{Ccl} 2$ and its receptor were higher in the ICB-sensitive models. CCL2 is a cytokine largely known for its involvement in the recruitment of CCR2+ monocytes from the bone marrow to other sites in the body where they differentiate into macrophages ${ }^{58,59}$. Additionally, CCL2 has been shown to recruit monocytes and macrophages to breast tumors and to facilitate breast cancer metastasis. ${ }^{60,61}$ The CCL2/CCR2 axis may represent an unique opportunity for anti-cancer therapy and work in this area is already being explored. ${ }^{62,63}$ The combination of CCL2 antagonism with anti-PD-1 has demonstrated efficacy in some mouse models. $^{64}$

It is currently unclear if the absolute number of myeloid cells within the TME or the proportion of myeloid cells of total CD45 immune cells is a more important factor for the efficacy of ICB. The data here suggest that the former is a stronger predictor of response, and that the phenotype might not be as critical since the ratio of M1:M2 macrophages was higher in the ICBresistance models (albeit lower absolute numbers of myeloid cells). Further work to understand TAM phenotype should be carefully noted by their function, signaling pathways and expression of extracellular markers. The functional activity of T-cells depends largely on the expression of co-stimulatory molecules, peptide-MHC complexes, MHC class I molecules, and expression of checkpoint markers (PD-1 and CTLA4). ${ }^{54}$ Additionally, T-cells secrete cytokines to promote a differential effector function. Activated T-cells (Th1 type) can secrete IL-2, TNFa and IFNy, which in turn induce cytotoxic function of CD8+ T-cells and promote phagocytosis through co-stimulatory markers on macrophages and other antigen presenting cells (CD40, CD86, and CD80). ${ }^{65,66}$ In contrast, secretion of IL-4 - $6-10-13$ by Th2 CD4+ T-cells can promote T-cell anergy and inhibit the activation of CTLs. ${ }^{40}$ Tregs are responsible for dampening the CTL responses and are characterized by expression of the transcription factor FoxP3. ${ }^{67}$ Tregs correlate with poor prognosis in a variety of epithelial tumor types possibly as a result of dampening T-cell immunity in response to cancer associated antigens. ${ }^{68,69}$ The most sensitive model (1E6) to ICB had a the lowest frequency of Tregs (Fig. 3a,g), but did not find a pattern of ICB response related to CTL numbers or proportions, as the autochthonous model had the highest frequency but was resistant to ICB. We did observe a correlation between baseline PD-L1 expression of myeloid cells (Fig. 4f) but not tumor cells (Fig. 4g) and response to ICB. This is an important observation seeing as inclusion criteria for some ICB treatment and/or clinical trials can include assessment of PD-L1 expression (NCT03258788, NCT02536794). We also noted by NanoString gene expression analysis the $1 \mathrm{E} 6$ tumors had elevated levels of both CD274 (PD-L1) and CTLA-4; which corresponds with the response to anti-CTLA-4 and anti-PD-L1 monotherapy (Fig. 5e). A limitation 
to this work is that the TME was not assessed after ICB, which may reveal additional changes that correlate with response to therapy.

\section{Conclusion}

The evasion of immune surveillance is a challenge in breast ICB therapy that warrants further investigation. Mechanistic understanding of how the TME promotes tumor progression will be critical to understand which cell populations play the most influential role in promoting an immune escape. Overall, we found that by immunophenotyping the three syngeneic models compared to the autochthonous model provided valuable insights for sensitivity to anti-CTLA4 and anti-PD-L1 therapy. We've uncovered that innate immunity and antigen processing may play a vital role in determining response to checkpoint blockade. Further work to characterize the signals within the TME that promote immune evasion will be vital to advancing checkpoint blockade therapy for the treatment of breast cancer. The four models of murine breast cancer presented here, of which 2 are ICB-sensitive and 2 are ICB-resistant, represent a unique opportunity to further interrogate biomarkers of response to ICB. 


\section{Figure Legends}

Figure 1. The number of cancer cells inoculated in pre-clinical models influences tumor growth kinetics as well as the tumor microenvironment.

a. Tumors from the autochthonous MMTV-PyMT model were harvested and single cell suspensions were generated. Cells (1E6, 1E5 or 1E4) were injected into mammary fat pad number 4 of FVB/NJ wild type mice. When the tumors reached $100 \mathrm{~mm}^{3}$, mice were randomized into an experimental group. b. Tumor volumes were measured every 3-4 days until individual tumors reached $100 \mathrm{~mm}^{3}$; tumor volumes are plotted as average tumor burden \pm SEM. c. Analysis comparing percent of CD45+ cells of total viable cells (left) and representative as pie charts (right). d. Analysis comparing percent of $\mathrm{CD}^{+}, \mathrm{CD} 11 \mathrm{~b}^{+}$, and other $\mathrm{CD} 45^{+}$cells of total viable cells. e.

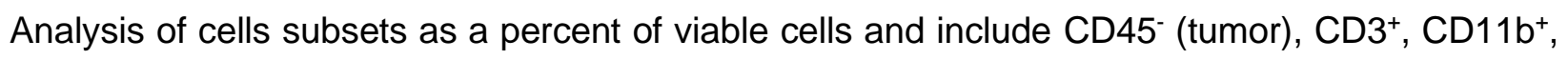
other immune (CD3-CD11 b-CD45 $\left.{ }^{+}\right)$populations. $\mathbf{f}$. Ratio of $C D 3^{+}$to $C D 11 b^{+}$out of viable cells. $\mathbf{g}$. Regression analysis of $\mathrm{CD}^{+}$as a variable of time for tumor to reach $100 \mathrm{~mm}^{3}$. Graphs show mean $\pm S E M$ from at least two independent experiments with at least three mice per group. Each data point represents an individual mouse. ${ }^{*} P<0.05,{ }^{* *} P<0.01,{ }^{* *} P<0.001$

Figure 2. Tumor infiltrating leukocyte populations differ significantly in the breast tumor models.

Tumors from the autochthonous MMTV-PyMT model were harvested and single cell suspensions were generated. Cells (1E6, $1 \mathrm{E} 5$ or 1E4) were injected into mammary fat pad number 4 of FVB/NJ wild type mice. When the tumors reached $100 \mathrm{~mm}^{3}$, tumors were obtained and processed into single cell suspension for immunophenotyping by flow cytometry. a. Analysis comparing absolute number of lymphocytes $\left(C D 3^{+}\right)$and lymphocyte subsets $\left(C D 8^{+}\right.$and $\left.C D 4^{+}\right)$per gram of tissue. b. Analysis comparing frequency of lymphocytes $\left(C D 3^{+}\right)$and lymphocyte subsets $\left(C D 4^{+}\right.$and $\left.C D 8^{+}\right)$ out of total $\mathrm{CD}_{4} 5^{+}$cells. c. Analysis comparing absolute number of myeloid cells (CD11 $\left.\mathrm{b}^{+}\right)$and myeloid cell subsets $\left(\mathrm{F} 4 / 80^{+}\right.$and $\left.\mathrm{Gr} 1^{+} \mathrm{CD} 11 \mathrm{~b}^{+}\right)$per gram of tissue. d. Analysis of myeloid cell 
subsets as a percent of total CD45 ${ }^{+}$cells. Graphs show mean \pm SEM from at least two independent experiments with at least three mice per group. Each data point represents an individual mouse. Unpaired two tailed t-test. ${ }^{\star} P<0.05,{ }^{\star *} P<0.01,{ }^{* \star} P<0.001,{ }^{* \star *} P<0.001$.

Figure 3. T-cell immune subsets differ significantly between the breast tumor models.

Tumors from the autochthonous MMTV-PyMT model were harvested and single cell suspensions were generated. Cells (1E6, 1E5 or 1E4) were injected into mammary fat pad number 4 of FVB/NJ wild type mice. When the tumors reached $100 \mathrm{~mm}^{3}$, tumors were obtained and processed into single cell suspension for immunophenotyping by flow cytometry. a-d. Analysis of T-cell subsets as a percent of $\mathrm{CD}^{+}$cells and include: a. T-regulatory cells $\left(\mathrm{FoxP} 3^{+} \mathrm{CD} 4^{+}\right.$), b. FoxP3 ${ }^{-} \mathrm{CD} 4^{+}$, $\mathbf{c}$. $\mathrm{CD}^{+}$, and d. other $\mathrm{CD}^{+}$cells. e. Analysis of lymphocyte cells subsets as a percent of viable cells represented as pie charts. f. The ratio of $\mathrm{CD}^{+}$to $\mathrm{CD}^{+}$out of total lymphocytes. g. Analysis of $\mathrm{T}$ regulatory cells represented by FoxP3 ${ }^{+}$of $\mathrm{CD}^{+}$cells. $\mathbf{h}$. The ratio of CTLs represented by GZB ${ }^{+}$ of $\mathrm{CD8}^{+}$. i. The ratio of CTLs to T-regulatory cells. Graphs show mean \pm SEM from at least two independent experiments with at least three mice per group. Each data point represents an individual mouse. Unpaired two tailed t-test. ${ }^{\star} P<0.05,{ }^{* *} P<0.01,{ }^{* * *} P<0.001,{ }^{* * *} P<0.001$.

\section{Figure 4. Myeloid immune cell subsets differ significantly breast tumor models.}

Tumors from the autochthonous MMTV-PyMT model were harvested and single cell suspensions were generated. Cells (1E6, 1E5 or 1E4) were injected into mammary fat pad number 4 of FVB/NJ wild type mice. When the tumors reached $100 \mathrm{~mm}^{3}$, tumors were obtained and processed into single cell suspension for immunophenotyping by flow cytometry. a-d. Analysis of cells subsets as a percent of myeloid cells $\left(\mathrm{CD} 11 b^{+}\right)$include a. MDSC $\left(\mathrm{GR} 1^{+}\right)$b. PD-L1+F4/80+ c. PD-L1$\mathrm{F} 4 / 80^{+}$d. other $\mathrm{CD} 11 \mathrm{~b}^{+}$populations; e. Analysis of the myeloid cells subsets as a percent of viable cells include: $\mathbf{f}$. Analysis of PD-L1 expression on myeloid cells $\left(\mathrm{CD} 11 \mathrm{~b}^{+}\right)$and $\mathbf{g}$. cancer cells represented by CD45. h. Analysis of the ratio of lymphocytes expressing PD-1. i. Analysis of M1 
macrophage markers, $\mathrm{CD}_{40}{ }^{+}, \mathrm{CD} 80^{+}$, and $\mathrm{CD}_{6} 6^{+}$and an M2-like macrophage marker, $\mathrm{CD} 206^{+}$, as a ratio of total myeloid cells (CD11b+, top), and mature macrophages ( $\mathrm{F} 4 / 80^{+}$, bottom). Graphs show mean $\pm S E M$ from at least two independent experiments with at least three mice per group. Each data point represents an individual mouse. Unpaired two tailed t-test. ${ }^{*} P<0.05$, ${ }^{* \star} P<0.01$, ${ }^{* * *} P<0.001,{ }^{* * *} P<0.001$.

\section{Figure 5. The breast tumor models have distinct immune transcriptional signatures.}

Tumors from the autochthonous MMTV-PyMT model were harvested and single cell suspensions were generated. Cells (1E6, 1E5 or 1E4) were injected into mammary fat pad number 4 of FVB/NJ wild type mice. When the tumors reached $100 \mathrm{~mm}^{3}$, tumors were obtained, and RNA was extracted from tumors to assess transcriptional changes. The NanoString PanCancer Immune Profiling Panel was used to analyze gene expression patterns related to the immune cell compartment of the tumors. a. A heatmap of all cell-type scores are shown. b. Quantification of cell types scores of immune cells, T-cells, and macrophages are shown. c. Hierarchically clustered heatmap showing the pathway scores across the four models. Yellow and blue

represent up and downregulated scores, respectively. d. Signature scores from the following pathways are highlighted: inflammation, adaptive immunity, T-cell function, innate immunity, dendritic cell functions, macrophage functions, MHC, and antigen processing. e. Normalized log2 counts are shown for the following transcripts: Cd274 (PD-L1), Ctla4, Ccr2, and Ccl2. Graphs show mean \pm Standard Deviation with 4-5 mice per group. Each data point represents an individual mouse. Unpaired two tailed t-test. ${ }^{\star} P<0.05,{ }^{* *} P<0.01,{ }^{* \star *} P<0.001,{ }^{* \star * *} P<0.001$.

Figure 6. The MMTV-PyMT syngeneic models respond differently to immune checkpoint blockade. 
Tumors from the autochthonous MMTV-PyMT model were harvested and single cell suspensions were generated. Cells (1E6, $1 \mathrm{E} 5$ or $1 \mathrm{E} 4)$ were injected into mammary fat pad number 4 of FVB/NJ wild type mice. When the tumors reached $100 \mathrm{~mm}^{3}$, mice were randomized into four treatment groups: Vehicle (IgG2B), anti-PD-L1, anti-CTLA-4, and the combination of anti-PD-L1 and antiCTLA-4. Tumor volumes were measured and plotted as mean total tumor burden \pm SEM (left) and survival analysis of mice are shown (right). Shown is a representative experiment of two individual experiments $n=3-7 /$ treatment groups for the $1 \mathrm{E} 6$ (a), $1 \mathrm{E} 5$ (b), $1 \mathrm{E} 4$ (c) and transgenic models (d). Two-way ANOVA multiple comparisons and Gehan-Breslow-Wilcoxon tests were performed. ${ }^{*} P<0.05,{ }^{* *} P<0.01,{ }^{* *} P<0.001,{ }^{* * *} P<0.001$.

Figure 7. Summary slide of cellular immune components that correlate with response to immunotherapy.

a. Key characteristics of immune cell subsets are summarized in the table and grouped by T-cells, myeloid cells, where "+" indicates significant changes, and blue and red represent significant increase and decrease expression, respectively, compared to the MMTV-PyMT model. Response to immune checkpoint blockade is also shown where blue and red represent significant increase and decrease response, respectively, compared to the vehicle control. b. Schematic representing the 4 tumor models and their immune composition. Figure created using biorender.com.

\section{Supplemental Figure 1. Immunophenotyping of cells used for generation of syngeneic murine models.}

Tumors from MMTV-PyMT autochthonous model were used to generate corresponding syngeneic tumor models. Cells injected into mice were first immunophenotyped by flow cytometry. Shown are three separate batches of tumor cells used for inoculation. a. Gating strategy for flow cytometry b. Immune cell composition as a percent out of viable cells. c. Frequency of immune cell populations out of $\mathrm{CD} 45^{+}$cells. Graphs show mean \pm SEM. 


\section{Supplemental Figure 2. Example of FACs gating strategy.}

a. Gating strategy for flow cytometry.

\section{Supplemental Figure 3. Ratio of anti-tumor to pro-tumor macrophages.}

Tumors from the autochthonous MMTV-PyMT model were harvested and single cell suspensions were generated. Cells (1E6, 1E5 or 1E4) were injected into mammary fat pad number 4 of FVB/NJ wild type mice. When the tumors reached $100 \mathrm{~mm}^{3}$, tumors were obtained and processed into single cell suspension for immunophenotyping by flow cytometry to identify the ratio of classically activated macrophages $\mathrm{CD}_{40}{ }^{+}(\mathbf{a}), \mathrm{CD} 80^{+}(\mathbf{b})$, and $\mathrm{CD} 86^{+}$(c) of $\mathrm{F} 4 / 80^{+}$to alternatively activated macrophages indicated by the mannose receptor, CD206 + of F4/80 + cells. Each data point represents an individual mouse. Graphs show mean \pm SEM. ${ }^{\star} P<0.05,{ }^{* \star} P<0.01,{ }^{* \star *} P<0.001,{ }^{* \star \star *}$ $P<0.001$.

\section{Supplemental Figure 4. QC of mRNA expression analysis.}

Tumors from the autochthonous MMTV-PyMT model were harvested and single cell suspensions were generated. Cells (1E6, 1 E5 or 1E4) were injected into mammary fat pad number 4 of FVB/NJ wild type mice. When the tumors reached $100 \mathrm{~mm}^{3}$, RNA was extracted from tumors and NanoString analysis was performed. Data was analyzed using the Advanced Analysis 2.0 Module of the nSolver ${ }^{T M}$ software (NanoString Technologies). The geNorm algorithm was used for selecting housekeeping genes and samples were normalized against positive controls. a. Normalization results shows consistency among selected housekeeping genes according to geNorm. b. Mean gene expression plotted versus variance. Housekeeping genes used in normalization are highlighted. c. Principle component analysis scored across the four models. The first two principal components explain $36 \%$ and $14 \%$ of variance respectively. d. Unsupervised hierarchical clustering was performed on all normalized transcripts above 
background. Orange cells indicate higher than average expression; blue cells indicate lower than average expression. e. A summary of cell types scores are shown across the four groups. e Quantification of cell types scores for neutrophils, dendritic cells, natural killer cells, and natural killer CD56dim are shown. Each data point represents an individual mouse. Graphs show mean \pm SEM. Unpaired two tailed $t$-test. ${ }^{*} P<0.05,{ }^{* *} P<0.01,{ }^{* * *} P<0.001,{ }^{* * * *} P<0.001$.

\section{Supplemental Figure 5. Quantification of pathway analysis by NanoString.}

Signature scores from the NanoString pathways analysis are shown. Graphs show mean \pm SEM with 4-5 mice per group. Unpaired two tailed t-test. ${ }^{*} P<0.05,{ }^{* *} P<0.01,{ }^{* * *} P<0.001,{ }^{* * * *} P<0.001$.

\section{Supplemental Figure 6. Pathway analysis by NanoString.}

a. The heatmap summarizes the mRNA transcripts categorized by their respective pathway. Shown are the signature scoring differences of the syngeneic models compared to the transgenic model. Orange represents higher than average scores; blue represents lower than average scores. b-d. Volcano plots representing differential expression analysis of genes expressed in the 1E6 (top), 1E5 (middle), 1E4 model (bottom) compared to the transgenic model. Volcano plots show fold change $(\log 2)$ versus $\log _{10} p$-values. False discovery rate thresholds are shown ( $F D R<0.01, F D R<0.05, F D R<0.1$, and $F D R<0.5)$. e. Normalized log2 counts of transcripts related to the Antigen presentation pathway from the Nanostring pathways analysis are shown. $f$. Normalized log2 counts of transcripts related to the immune signaling pathway are shown. Graphs show mean \pm SEM with 4-5 mice per group. Unpaired two tailed $t$-test. ${ }^{\star} P<0.05,{ }^{* \star} P<0.01,{ }^{* * *}$ $P<0.001,{ }^{* * \star *} P<0.001$. 
Supplemental Figure 7. Syngeneic tumor treated with CTLA-4 and PD-L1 show significant differences in tumor fold change.

Mice of similar tumor burden from each syngeneic model and the autochthonous model were randomized into four treatment groups: IgG2B, PD-L1 inhibitor, CTLA-4 inhibitor, and the combination of PD-L1 and CTLA-4 inhibitors. Tumors were measured every 2-3 days. Tumor volumes were measured and plotted as mean total tumor burden \pm SEM and tumor burden fold change was calculated at day 14 for the 1E6 (a), 1E5 (b), 1 E4 (c) and autochthonous (d) models. Shown are a summary of two individual experiments $n=3-7 /$ treatment groups. Two-way ANOVA multiple comparisons was performed ${ }^{\star} P<0.05,{ }^{* \star} P<0.01,{ }^{* \star *} P<0.001,{ }^{\star \star \star *} P<0.001$.

\section{Castrillon et. al. Supplemental Figure 8. Comparison of predicted responders vs.} non-responders by NanoString analysis.

The NanoString data was reanalyzed grouping mice into predicted responders (1E6 and 1E5) and predicted non-responders (1E4 and autochthonous). a. Principle component analysis. b. Heatmap of immune cell scores. c. Cell types scores are shown across the two groups. Each data point represents an individual mouse. d. Heatmap of pathway analysis. e. Differential expression of responders vs non-responders with the Inflammation Score Probe Set shown in red. Graphs show mean \pm SEM. Unpaired two tailed $t$-test. ${ }^{*} P<0.05,{ }^{* *} P<0.01,{ }^{* *} P<0.001,{ }^{* * * *} P<0.001$. 


\section{Ethical Approval and Consent to participate}

All mice were maintained within the Dana-Farer Cancer Center (DFCI) and all experiments were conducted under The Institutional Animal Care and Use Committee (IACUC).

\section{Consent for publication}

$\mathrm{n} / \mathrm{a}$

\section{Availability of supporting data}

The datasets used and/or analyzed during the current study are available from the corresponding author on reasonable request.

\section{Competing interests}

J.L.G. is a consultant for Glaxo-Smith Kline (GSK), Array BioPharma, Codagenix and Verseau, and receives sponsored research support from GSK, Eli Lilly and Array BioPharma. E.A.M has served on SABs for Astra-Zeneca/Medimmune, Celgene, Genentech, Genomic Health, Merck, Peregrine Pharmaceuticals, SELLAS Lifescience, and Tapimmune and has clinical trial support to her former institution (MDACC) from astra-zeneca/Medimmune, EMDSerono, Galena Biopharma and Genentech as well as Genentech support to a SU2C grant, as well as sponsored Research Support to the laboratory from GSK and Eli Lilly. A.L. is a consultant for Astra-Zeneca, receives sponsored research support from Novartis, and serves on the scientific advisory board for Flash Therapeutics, Zentalis, and Dialectic. E.M. is an employee and stockholder of NanoString Technologies.

\section{Funding}

J.L.G is supported by a Career Enhancement Award through the National Institutes of Health (NIH) grant Dana-Farber/Harvard Cancer Center Breast Cancer SPORE 5P50CA168504 and a Komen Career Catalyst Award CCR18547597. A.L, E.A.M and J.L.G are supported through the Ludwig Center at Harvard.

\section{Authors contributions}

JAC, AKM, EC, EM and AS performed all experiments and analyzed and interpreted the data. JC, AKM and JLG write the manuscript. EAM, AL and JLG provided senior leadership. All authors read, edited and approved the final manuscript. 


\section{Acknowledgements}

The authors thank Suzan Lazo for her technical help setting up flow cytometry panels. The authors are grateful for the expertise and assistance from the Dana-Farber Animal Research Facility and Flow Cytometry Core. 


\section{References}

1 Waks, A. G. \& Winer, E. P. Breast Cancer Treatment: A Review. Jama 321, 288-300, doi:10.1001/jama.2018.19323 (2019).

2 Early Breast Cancer Trialists' Collaborative, G. et al. Relevance of breast cancer hormone receptors and other factors to the efficacy of adjuvant tamoxifen: patient-level meta-analysis of randomised trials. Lancet 378, 771-784, doi:10.1016/S0140-6736(11)60993-8 (2011).

3 Slamon, D. J. et al. Use of Chemotherapy plus a Monoclonal Antibody against HER2 for Metastatic Breast Cancer That Overexpresses HER2. New England Journal of Medicine 344, 783-792, doi:10.1056/nejm200103153441101 (2001).

4 Correa, C. et al. Accelerated Partial Breast Irradiation: Executive summary for the update of an ASTRO Evidence-Based Consensus Statement. Pract Radiat Oncol 7, 73-79, doi:10.1016/j.prro.2016.09.007 (2017).

5 O'Shaughnessy, J. Extending survival with chemotherapy in metastatic breast cancer. Oncologist 10 Suppl 3, 20-29, doi:10.1634/theoncologist.10-90003-20 (2005).

6 Emens, L. A. Breast Cancer Immunotherapy: Facts and Hopes. Clin Cancer Res 24, 511-520, doi:10.1158/1078-0432.CCR-16-3001 (2018).

7 Das, R. et al. Combination therapy with anti-CTLA-4 and anti-PD-1 leads to distinct immunologic changes in vivo. J Immunol 194, 950-959, doi:10.4049/jimmunol.1401686 (2015).

8 Pardoll, D. M. The blockade of immune checkpoints in cancer immunotherapy. Nat Rev Cancer 12, 252-264, doi:10.1038/nrc3239 (2012).

9 Hodi, F. S. et al. Improved survival with ipilimumab in patients with metastatic melanoma. $N$ Engl J Med 363, 711-723, doi:10.1056/NEJMoa1003466 (2010).

10 Schadendorf, D. et al. Pooled Analysis of Long-Term Survival Data From Phase II and Phase III Trials of Ipilimumab in Unresectable or Metastatic Melanoma. J Clin Oncol 33, 1889-1894, doi:10.1200/JCO.2014.56.2736 (2015).

11 Tsao, H., Atkins, M. B. \& Sober, A. J. Management of cutaneous melanoma. N Engl J Med 351, 9981012, doi:10.1056/NEJMra041245 (2004).

12 Lipson, E. J. \& Drake, C. G. Ipilimumab: an anti-CTLA-4 antibody for metastatic melanoma. Clin Cancer Res 17, 6958-6962, doi:10.1158/1078-0432.CCR-11-1595 (2011).

13 Brahmer, J. R. et al. Safety and activity of anti-PD-L1 antibody in patients with advanced cancer. N Engl J Med 366, 2455-2465, doi:10.1056/NEJMoa1200694 (2012).

14 Powles, T. et al. A phase Ib study investigating the combination of everolimus and dovitinib in vascular endothelial growth factor refractory clear cell renal cancer. Eur J Cancer 50, 2057-2064, doi:10.1016/j.ejca.2014.04.021 (2014).

15 Baumeister, S. H., Freeman, G. J., Dranoff, G. \& Sharpe, A. H. Coinhibitory Pathways in Immunotherapy for Cancer. Annu Rev Immunol 34, 539-573, doi:10.1146/annurev-immunol032414-112049 (2016).

16 Postow, M. A., Callahan, M. K. \& Wolchok, J. D. Immune Checkpoint Blockade in Cancer Therapy. J Clin Oncol 33, 1974-1982, doi:10.1200/JCO.2014.59.4358 (2015).

17 Topalian, S. L., Drake, C. G. \& Pardoll, D. M. Immune checkpoint blockade: a common denominator approach to cancer therapy. Cancer Cell 27, 450-461, doi:10.1016/j.ccell.2015.03.001 (2015).

18 Vokes, E. E. et al. Nivolumab versus docetaxel in previously treated advanced non-small-cell lung cancer (CheckMate 017 and CheckMate 057): 3-year update and outcomes in patients with liver metastases. Ann Oncol 29, 959-965, doi:10.1093/annonc/mdy041 (2018).

19 Antonia, S. J. et al. Overall Survival with Durvalumab after Chemoradiotherapy in Stage III NSCLC. N Engl J Med 379, 2342-2350, doi:10.1056/NEJMoa1809697 (2018). 
Overman, M. J. et al. Nivolumab in patients with metastatic DNA mismatch repair-deficient or microsatellite instability-high colorectal cancer (CheckMate 142): an open-label, multicentre, phase 2 study. Lancet Oncol 18, 1182-1191, doi:10.1016/S1470-2045(17)30422-9 (2017).

21 Davis, A. A. \& Patel, V. G. The role of PD-L1 expression as a predictive biomarker: an analysis of all US Food and Drug Administration (FDA) approvals of immune checkpoint inhibitors. Journal for ImmunoTherapy of Cancer 7, 278, doi:10.1186/s40425-019-0768-9 (2019).

22 Wolchok, J. D. et al. Overall Survival with Combined Nivolumab and Ipilimumab in Advanced Melanoma. N Engl J Med 377, 1345-1356, doi:10.1056/NEJMoa1709684 (2017).

23 Postow, M. A., Callahan, M. K. \& Wolchok, J. D. Immune Checkpoint Blockade in Cancer Therapy. Journal of clinical oncology : official journal of the American Society of Clinical Oncology 33, 19741982, doi:10.1200/JCO.2014.59.4358 (2015).

24 Nanda, R. et al. Pembrolizumab in Patients With Advanced Triple-Negative Breast Cancer: Phase Ib KEYNOTE-012 Study. J Clin Oncol 34, 2460-2467, doi:10.1200/JCO.2015.64.8931 (2016).

25 Colli, L. M. et al. Burden of Nonsynonymous Mutations among TCGA Cancers and Candidate Immune Checkpoint Inhibitor Responses. Cancer Res 76, 3767-3772, doi:10.1158/00085472.CAN-16-0170 (2016).

26 Bertucci, F. et al. Genomic characterization of metastatic breast cancers. Nature 569, 560-564, doi:10.1038/s41586-019-1056-z (2019).

27 Cortés, J. et al. KEYNOTE-119: Phase III study of pembrolizumab (pembro) versus single-agent chemotherapy (chemo) for metastatic triple negative breast cancer (mTNBC). Annals of Oncology 30, v859-v860, doi:10.1093/annonc/mdz394.010 (2019).

28 Adams, S. et al. Abstract PD6-10: KEYNOTE-086 cohort B: Pembrolizumab monotherapy for PDL1-positive, previously untreated, metastatic triple-negative breast cancer (mTNBC). Cancer Research 78, PD6-10-PD16-10, doi:10.1158/1538-7445.sabcs17-pd6-10 (2018).

29 Emens, L. A. et al. Long-term Clinical Outcomes and Biomarker Analyses of Atezolizumab Therapy for Patients With Metastatic Triple-Negative Breast Cancer: A Phase 1 Study. JAMA Oncol 5, 7482, doi:10.1001/jamaoncol.2018.4224 (2019).

30 Adams, S. et al. Phase 2 study of pembrolizumab (pembro) monotherapy for previously treated metastatic triple-negative breast cancer (mTNBC): KEYNOTE-086 cohort A. Journal of Clinical Oncology 35, 1008-1008, doi:10.1200/JCO.2017.35.15_suppl.1008 (2017).

31 Schmid, P. et al. Atezolizumab and Nab-Paclitaxel in Advanced Triple-Negative Breast Cancer. $N$ Engl J Med 379, 2108-2121, doi:10.1056/NEJMoa1809615 (2018).

32 Emens, L. et al. Abstract GS1-04: IMpassion130: Efficacy in immune biomarker subgroups from the global, randomized, double-blind, placebo-controlled, phase III study of atezolizumab + $<$ em>nab</em>-paclitaxel in patients with treatment-naïve, locally advanced or metastatic triplenegative breast cancer. Cancer Research 79, GS1-04-GS01-04, doi:10.1158/1538-7445.Sabcs18gs1-04 (2019).

33 Rimm, D. L. et al. Reanalysis of the NCCN PD-L1 companion diagnostic assay study for lung cancer in the context of PD-L1 expression findings in triple-negative breast cancer. Breast Cancer Res 21, 72, doi:10.1186/s13058-019-1156-6 (2019).

34 Luen, S. J. et al. Tumour-infiltrating lymphocytes in advanced HER2-positive breast cancer treated with pertuzumab or placebo in addition to trastuzumab and docetaxel: a retrospective analysis of the CLEOPATRA study. Lancet Oncol 18, 52-62, doi:10.1016/S1470-2045(16)30631-3 (2017).

35 Gil Del Alcazar, C. R. et al. Immune Escape in Breast Cancer During In Situ to Invasive Carcinoma Transition. Cancer Discov 7, 1098-1115, doi:10.1158/2159-8290.Cd-17-0222 (2017).

36 Walunas, T. L. et al. CTLA-4 can function as a negative regulator of T cell activation. Immunity 1 , 405-413, doi:10.1016/1074-7613(94)90071-x (1994). 

cancer model provides a reliable model for human diseases. Am J Pathol 163, 2113-2126, doi:10.1016/S0002-9440(10)63568-7 (2003).

38 Hollern, D. P. \& Andrechek, E. R. A genomic analysis of mouse models of breast cancer reveals molecular features of mouse models and relationships to human breast cancer. Breast Cancer Res 16, R59, doi:10.1186/bcr3672 (2014).

39 Guerriero, J. L. et al. Class Ila HDAC inhibition reduces breast tumours and metastases through anti-tumour macrophages. Nature 543, 428-432, doi:10.1038/nature21409 (2017).

40 DeNardo, D. G. et al. CD4(+) T cells regulate pulmonary metastasis of mammary carcinomas by enhancing protumor properties of macrophages. Cancer Cell 16, 91-102, doi:10.1016/j.ccr.2009.06.018 (2009).

41 Qian, B. Z. et al. CCL2 recruits inflammatory monocytes to facilitate breast-tumour metastasis. Nature 475, 222-225, doi:10.1038/nature10138 (2011).

42 Ruffell, B. et al. Leukocyte composition of human breast cancer. Proc Natl Acad Sci U S A 109, 2796-2801, doi:10.1073/pnas.1104303108 (2012).

43 Biswas, S. K. \& Mantovani, A. Macrophage plasticity and interaction with lymphocyte subsets: cancer as a paradigm. Nat Immunol 11, 889-896, doi:10.1038/ni.1937 (2010).

44 Solinas, G., Germano, G., Mantovani, A. \& Allavena, P. Tumor-associated macrophages (TAM) as major players of the cancer-related inflammation. J Leukoc Biol 86, 1065-1073, doi:10.1189/jlb.0609385 (2009).

45 Qian, B. Z. \& Pollard, J. W. Macrophage diversity enhances tumor progression and metastasis. Cell 141, 39-51, doi:10.1016/j.cell.2010.03.014 (2010).

46 Schmittnaegel, M. et al. Dual angiopoietin-2 and VEGFA inhibition elicits antitumor immunity that is enhanced by PD-1 checkpoint blockade. Sci Transl Med 9, doi:10.1126/scitranslmed.aak9670 (2017).

47 Messenheimer, D. J. et al. Timing of PD-1 Blockade Is Critical to Effective Combination Immunotherapy with Anti-OX40. Clin Cancer Res 23, 6165-6177, doi:10.1158/1078-0432.CCR-162677 (2017).

48 Vandesompele, J. et al. Accurate normalization of real-time quantitative RT-PCR data by geometric averaging of multiple internal control genes. Genome Biol 3, RESEARCH0034, doi:10.1186/gb-2002-3-7-research0034 (2002).

49 Tomfohr, J., Lu, J. \& Kepler, T. B. Pathway level analysis of gene expression using singular value decomposition. BMC Bioinformatics 6, 225, doi:10.1186/1471-2105-6-225 (2005).

50 Danaher, P. et al. Gene expression markers of Tumor Infiltrating Leukocytes. J Immunother Cancer 5, 18, doi:10.1186/s40425-017-0215-8 (2017).

51 Damuzzo, V. et al. Complexity and challenges in defining myeloid-derived suppressor cells. Cytometry. Part B, Clinical cytometry 88, 77-91, doi:10.1002/cyto.b.21206 (2015).

52 Veglia, F., Perego, M. \& Gabrilovich, D. Myeloid-derived suppressor cells coming of age. Nature Immunology 19, 108-119, doi:10.1038/s41590-017-0022-x (2018).

53 Allen, E. et al. Combined antiangiogenic and anti-PD-L1 therapy stimulates tumor immunity through HEV formation. Sci Transl Med 9, eaak9679, doi:10.1126/scitranslmed.aak9679 (2017).

54 Guerriero, J. L. in International Review of Cell and Molecular Biology (Academic Press, 2018).

55 Simpson, T. R. et al. Fc-dependent depletion of tumor-infiltrating regulatory T cells co-defines the efficacy of anti-CTLA-4 therapy against melanoma. J Exp Med 210, 1695-1710, doi:10.1084/jem.20130579 (2013).

56 Gul, N. et al. Macrophages eliminate circulating tumor cells after monoclonal antibody therapy. J Clin Invest 124, 812-823, doi:10.1172/jci66776 (2014). 
57 Kuang, D. M. et al. Activated monocytes in peritumoral stroma of hepatocellular carcinoma foster immune privilege and disease progression through PD-L1. J Exp Med 206, 1327-1337, doi:10.1084/jem.20082173 (2009).

58 Fuentes, M. E. et al. Controlled recruitment of monocytes and macrophages to specific organs through transgenic expression of monocyte chemoattractant protein-1. J Immunol 155, 57695776 (1995).

59 Kersten, K. et al. Mammary tumor-derived CCL2 enhances pro-metastatic systemic inflammation through upregulation of IL1 $\beta$ in tumor-associated macrophages. Oncoimmunology 6, e1334744e1334744, doi:10.1080/2162402X.2017.1334744 (2017).

60 Kitamura, T. et al. CCL2-induced chemokine cascade promotes breast cancer metastasis by enhancing retention of metastasis-associated macrophages. The Journal of experimental medicine 212, 1043-1059, doi:10.1084/jem.20141836 (2015).

61 Qian, B.-Z. et al. CCL2 recruits inflammatory monocytes to facilitate breast-tumour metastasis. Nature 475, 222-225, doi:10.1038/nature10138 (2011).

62 Lim, S. Y., Yuzhalin, A. E., Gordon-Weeks, A. N. \& Muschel, R. J. Targeting the CCL2-CCR2 signaling axis in cancer metastasis. Oncotarget 7, 28697-28710, doi:10.18632/oncotarget.7376 (2016).

63 Cassetta, L. \& Pollard, J. W. Targeting macrophages: therapeutic approaches in cancer. Nat Rev Drug Discov 17, 887-904, doi:10.1038/nrd.2018.169 (2018).

64 Wang, Y., Zhang, X., Yang, L., Xue, J. \& Hu, G. Blockade of CCL2 enhances immunotherapeutic effect of anti-PD1 in lung cancer. Journal of Bone Oncology 11, 27-32, doi:10.1016/j.jbo.2018.01.002 (2018).

65 Stout, R. D. \& Bottomly, K. Antigen-specific activation of effector macrophages by IFN-gamma producing (TH1) T cell clones. Failure of IL-4-producing (TH2) T cell clones to activate effector function in macrophages. J Immunol 142, 760-765 (1989).

66 Espinosa-Cueto, P., Magallanes-Puebla, A., Castellanos, C. \& Mancilla, R. Dendritic cells that phagocytose apoptotic macrophages loaded with mycobacterial antigens activate CD8 T cells via cross-presentation. PLoS One 12, e0182126, doi:10.1371/journal.pone.0182126 (2017).

67 Zou, W. Regulatory T cells, tumour immunity and immunotherapy. Nat Rev Immunol 6, 295-307, doi:10.1038/nri1806 (2006).

68 Curiel, T. J. et al. Specific recruitment of regulatory T cells in ovarian carcinoma fosters immune privilege and predicts reduced survival. Nat Med 10, 942-949, doi:10.1038/nm1093 (2004).

69 Kono, K. et al. CD4(+)CD25high regulatory T cells increase with tumor stage in patients with gastric and esophageal cancers. Cancer Immunol Immunother 55, 1064-1071, doi:10.1007/s00262-0050092-8 (2006). 

influences tumor growth kinetics as well as the tumor microenvironment.

a.

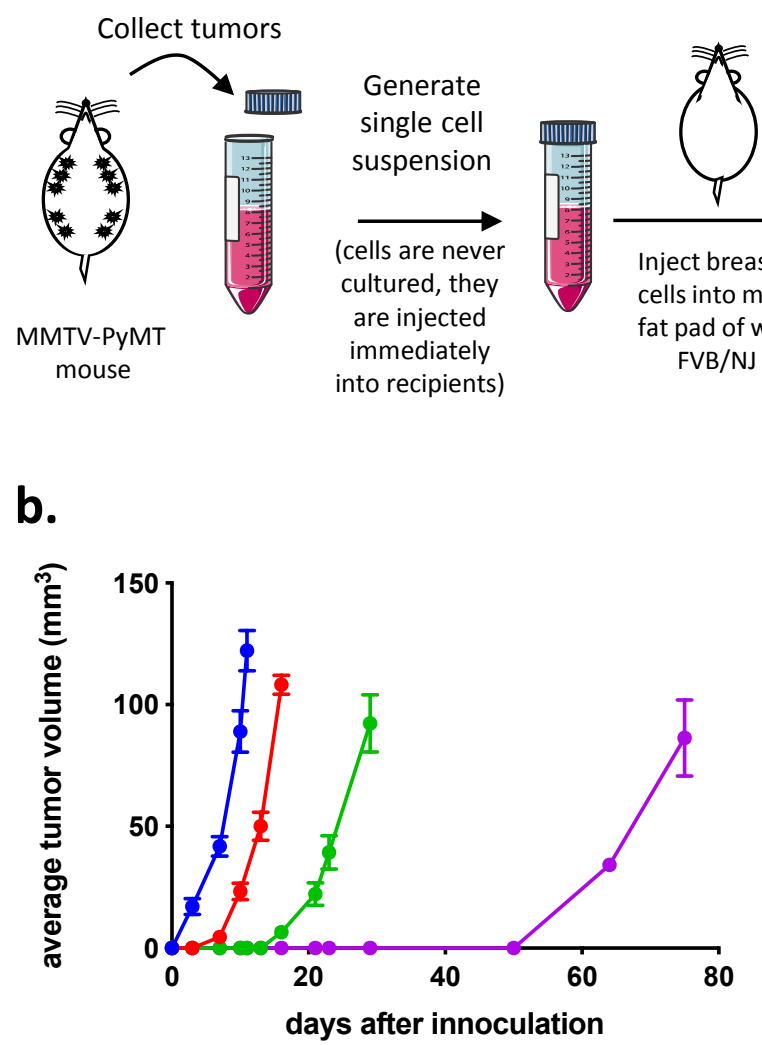

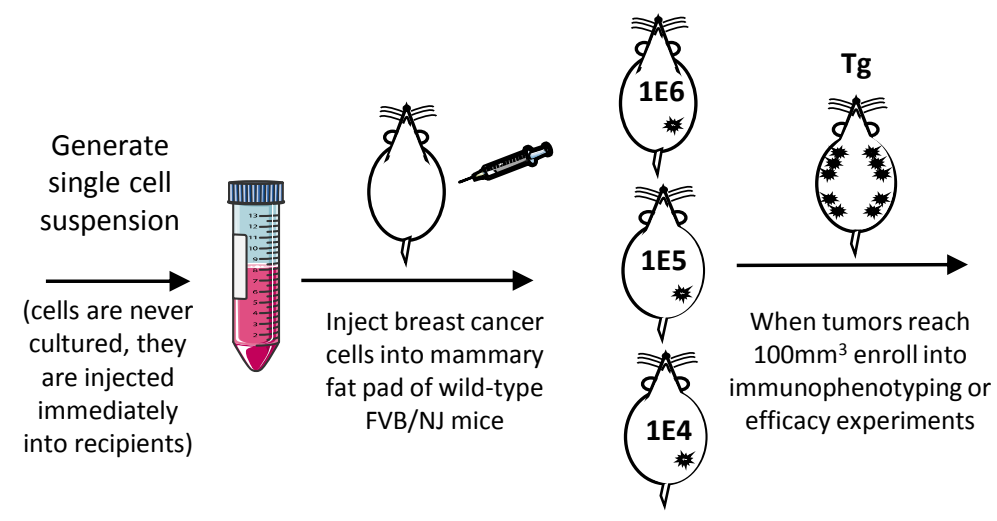

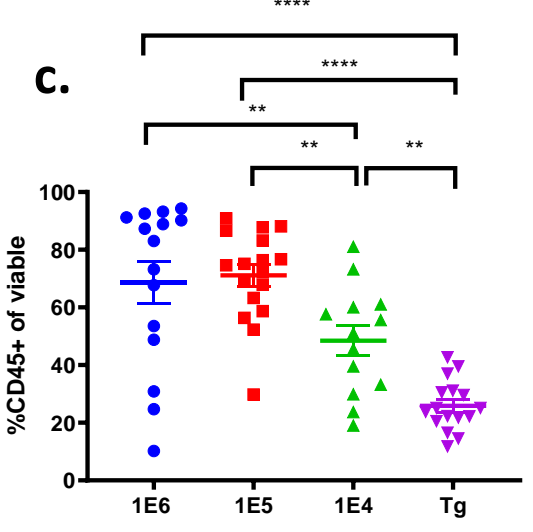

Immunophenotype by FACs:

1. Macrophage Panel

2. T-cell Panel

3. Checkpoint Marker Panel

Gene Expression:

Nanostring

Enroll into efficacy study:

1. Vehicle

2. Anti-CTLA4

3. Anti-PD-L1

4. Anti-CTLA4 + Anti-PD-L1

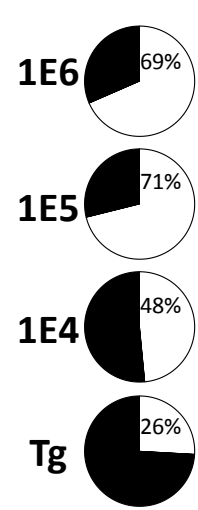

e.

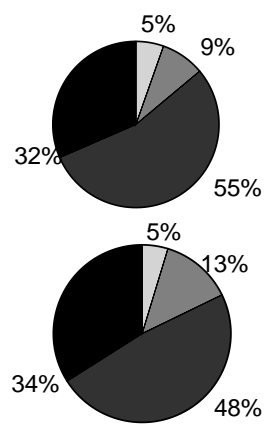

1E6
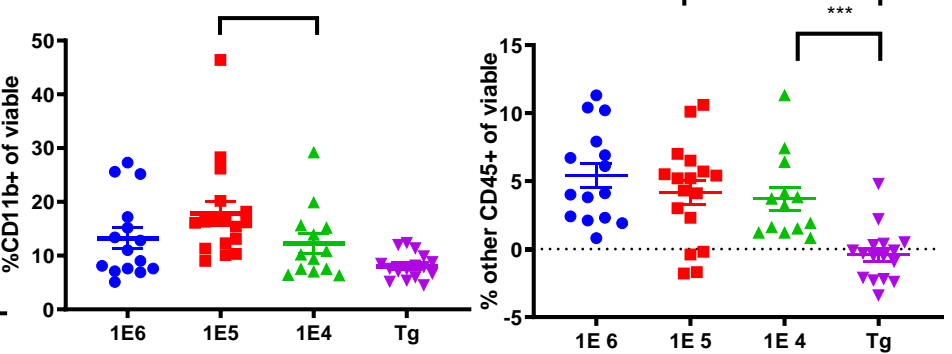

g.

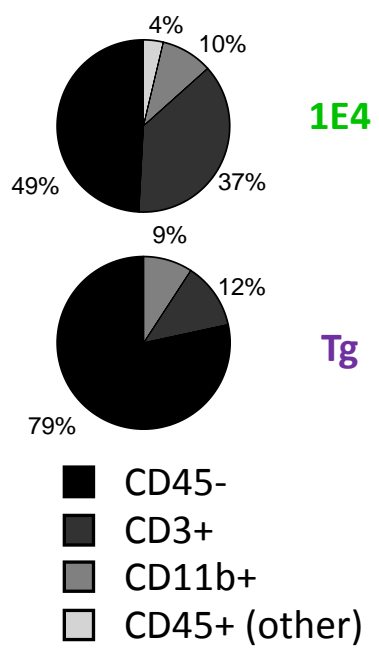



breast tumor models.

a. T-cells: of total (per gram of tumor tissue)

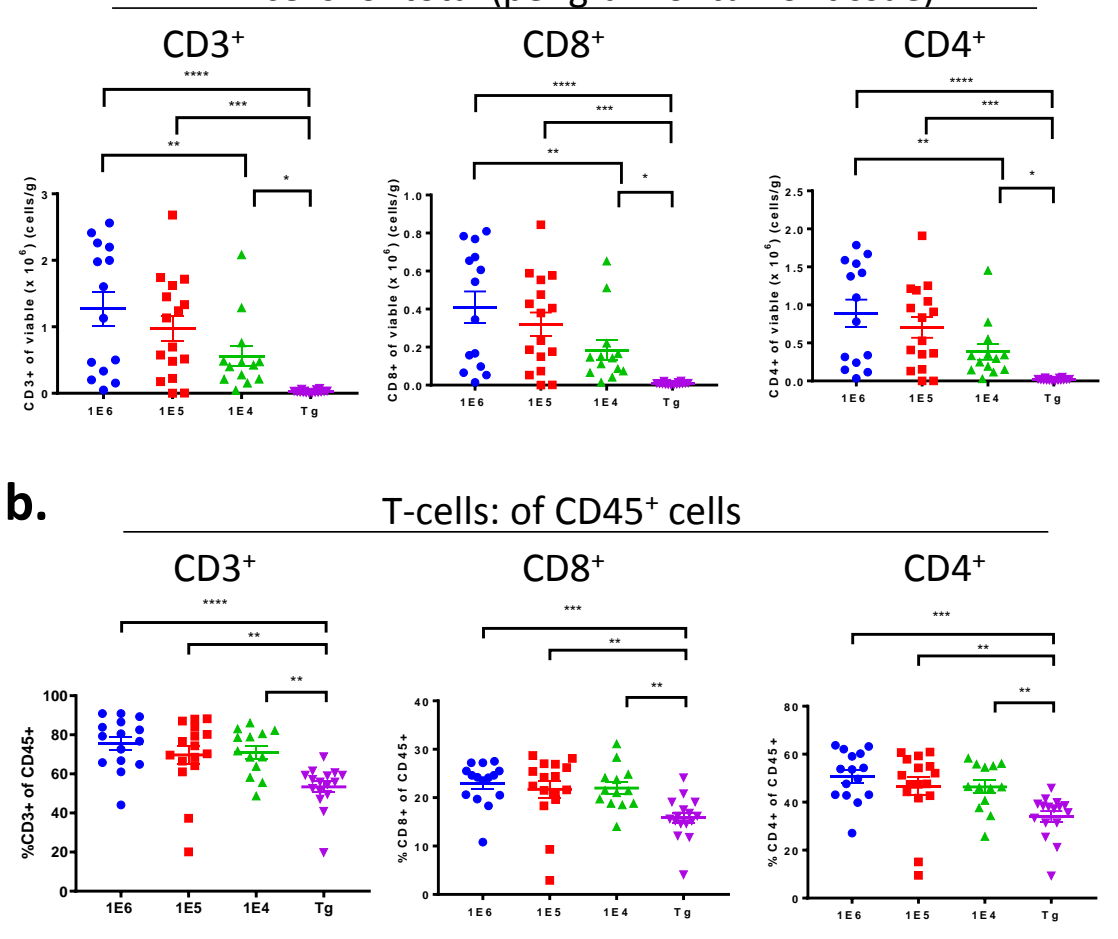

c.

Myeloid cells: of total (per gram of tumor tissue)
CD11b+
$\mathrm{F} 4 / 80^{+}$
Gr1+CD11 $b^{+}$
CD11b of CD45+
$\mathrm{F} 4 / 80^{+}$of $\mathrm{CD} 45^{+}$
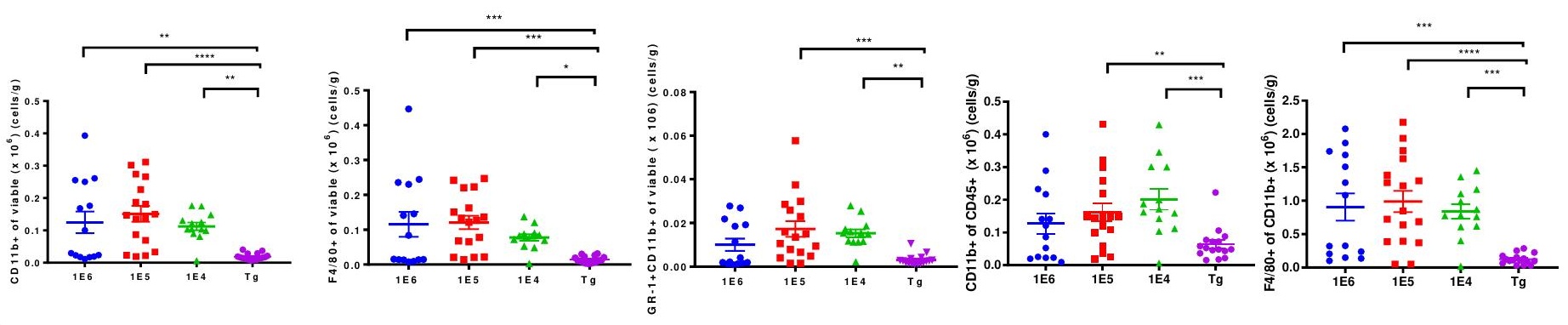

d.

Myeloid cells: of $\mathrm{CD} 45^{+}$cells

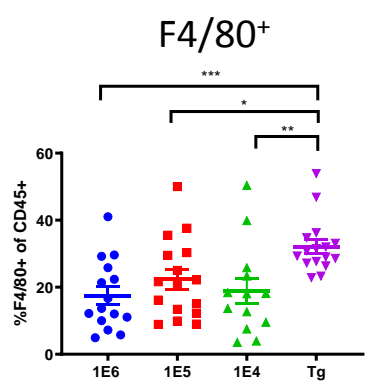

Gr1+CD11b+

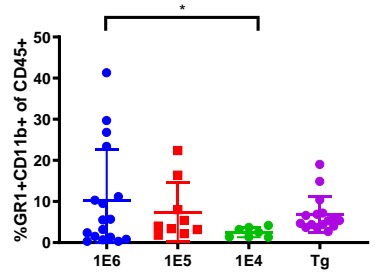

e.

$\mathrm{CD} 11 \mathrm{c}^{+}$
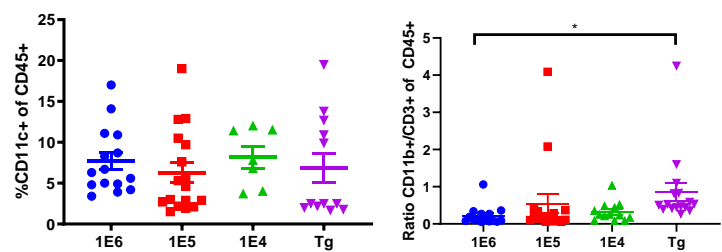

CD11b/CD3

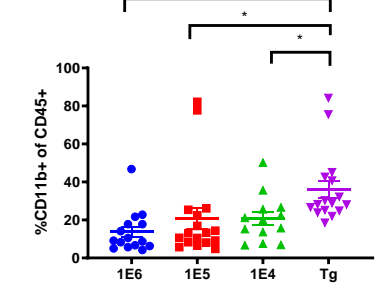


Castrillon et. al. Figure 3. T-cell immune subsets differ significantly between the breast tumor models.
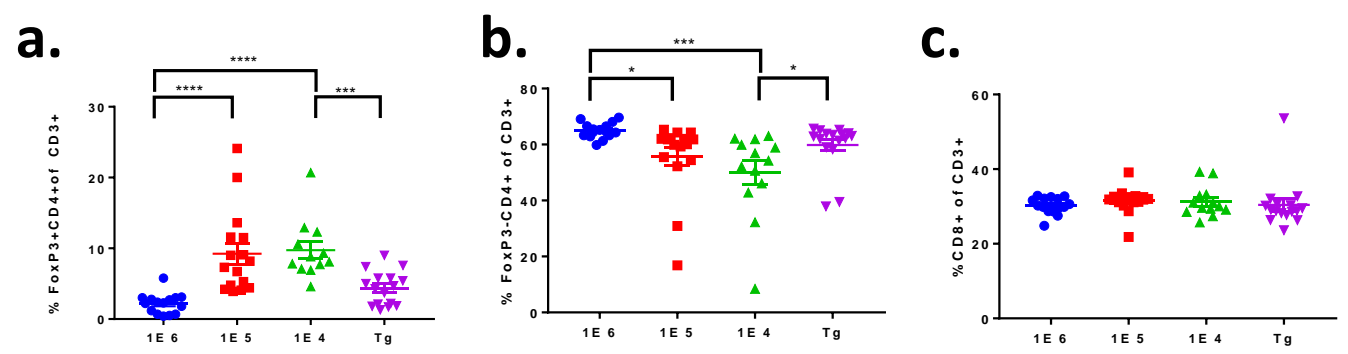

d.

e.

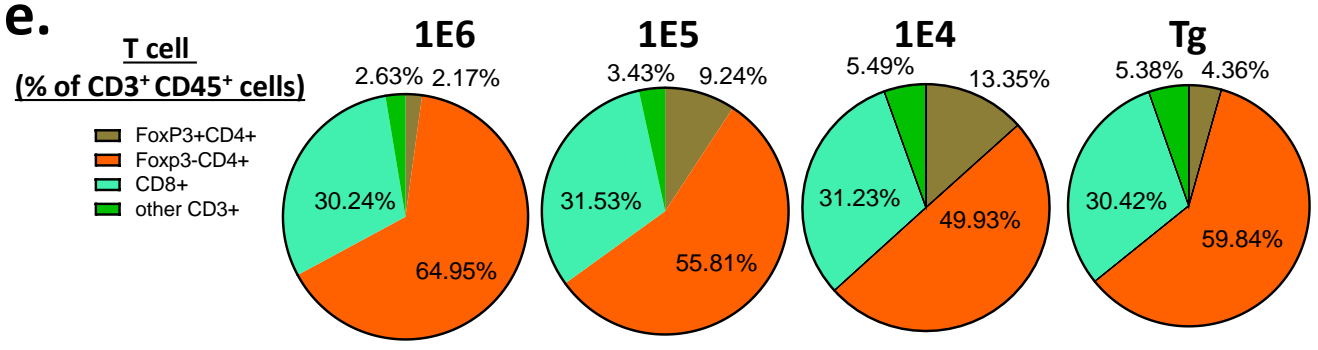

g.

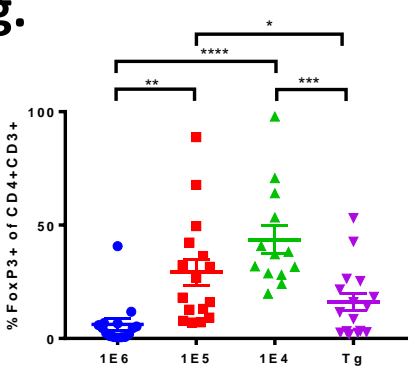

h.

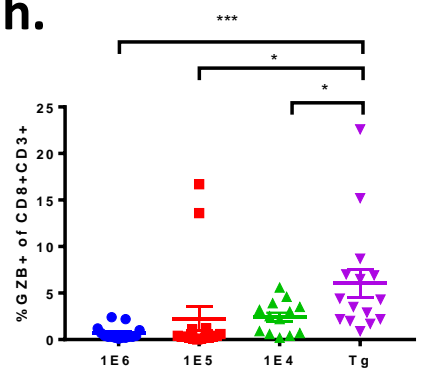

i.

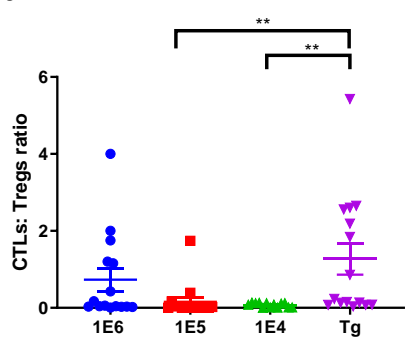

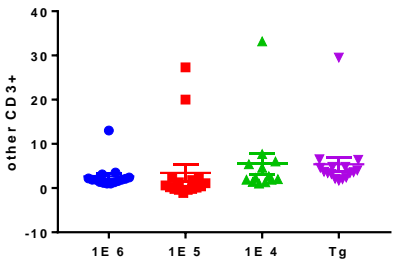

f.

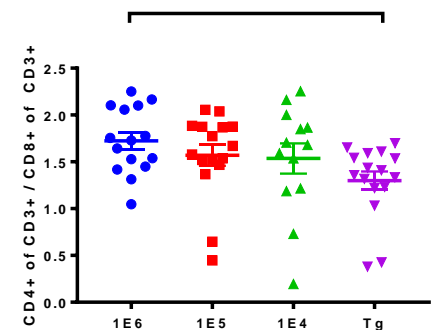


Castrillon et. al. Figure 4. Myeloid immune cell subsets differ significantly in the breast tumor models.
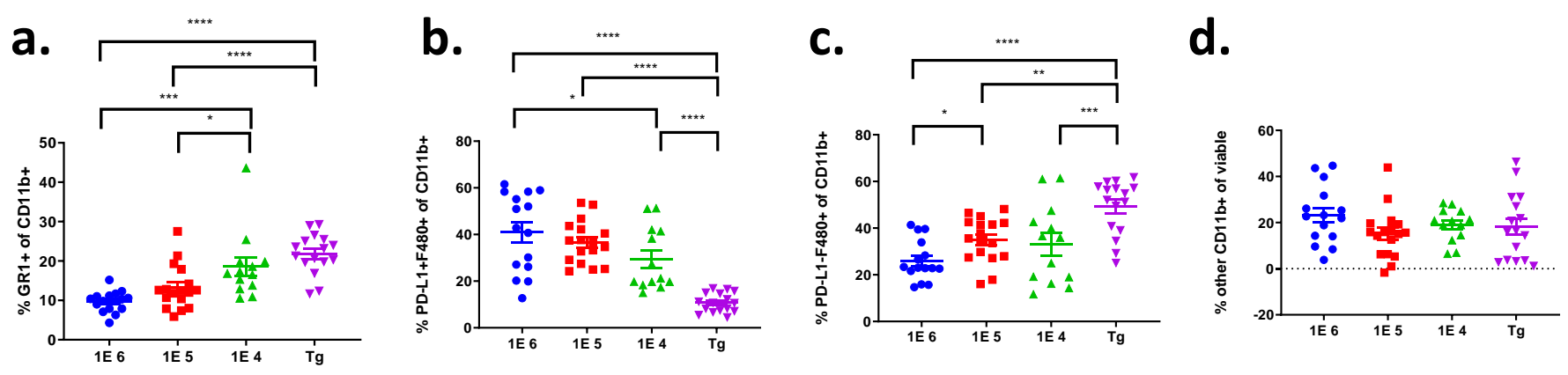

e.

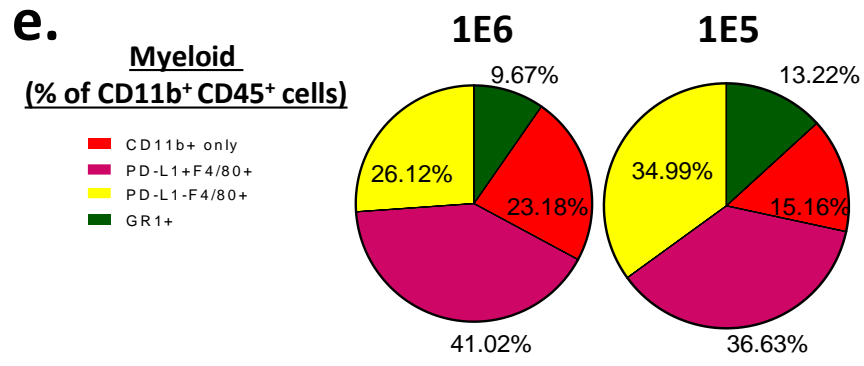

f.
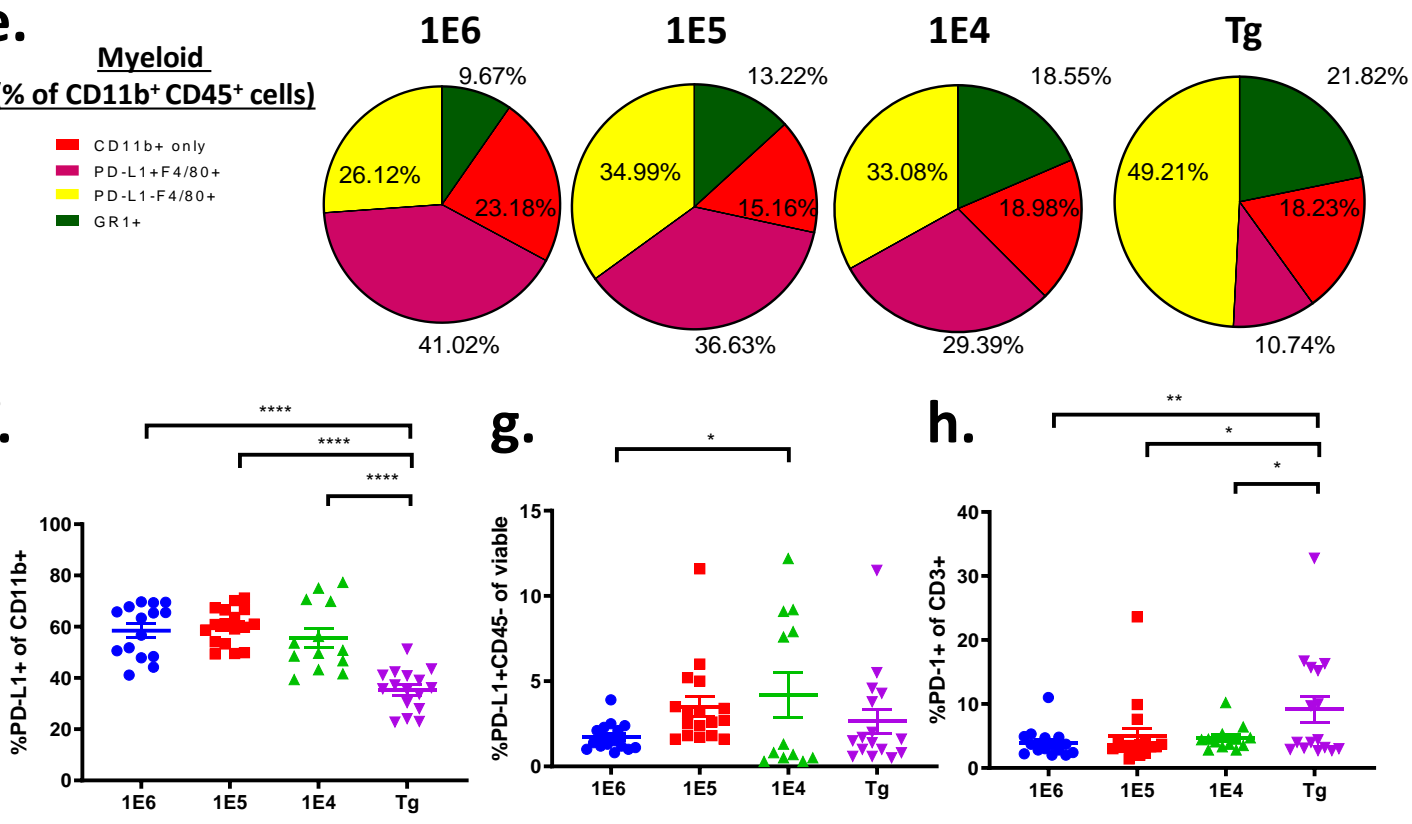

i.
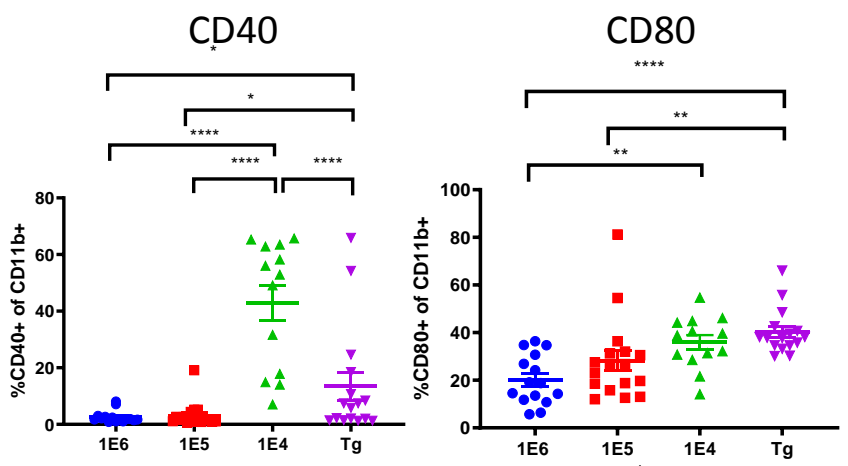

h.

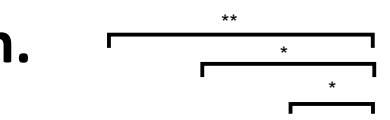

s.
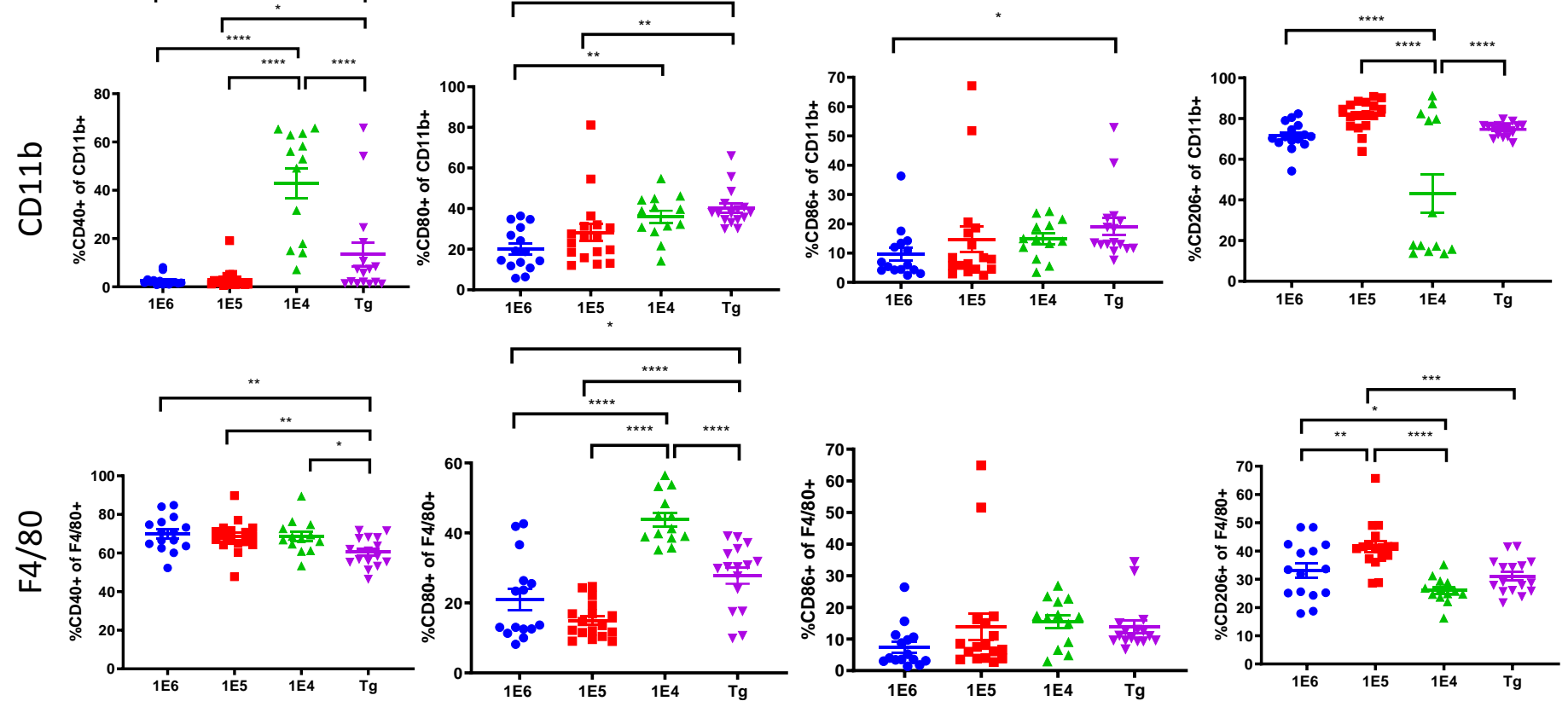
Castrillon et. al. Figure 5. The breast tumor models have distinct immune transcriptional signatures.

a.

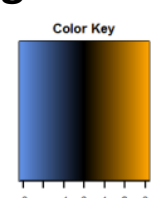

C.

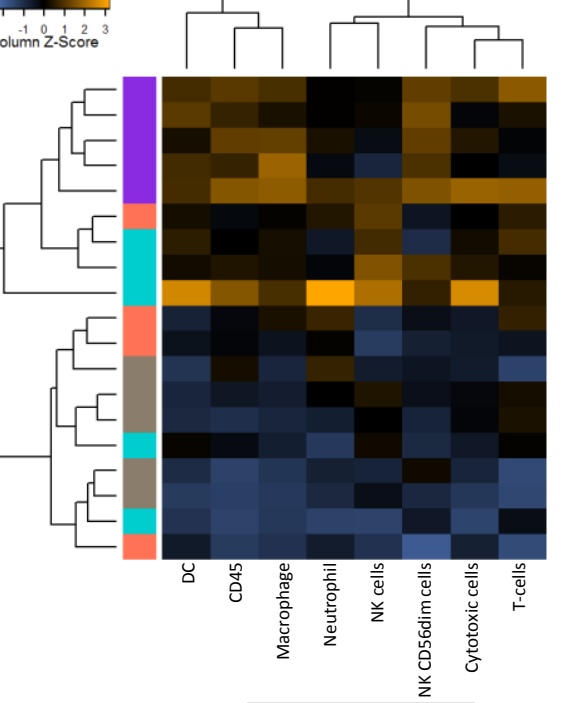

e

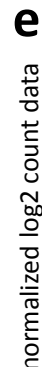

Cd274

Ctla4

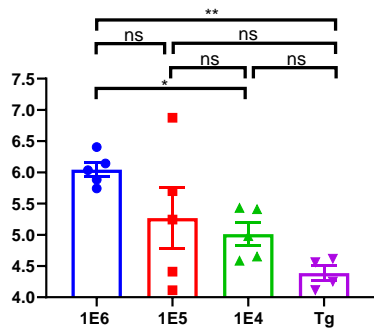

$1 \mathrm{E} 6$

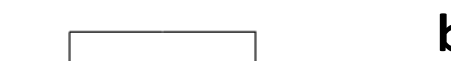

b.

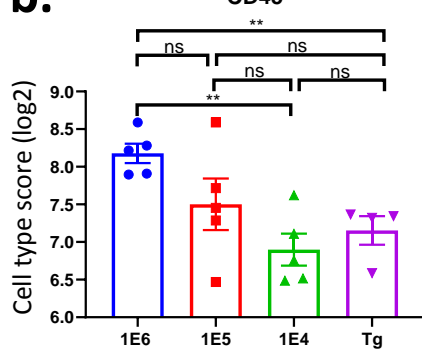

$1 \mathrm{E} 6$

1E5

1E4

$\mathrm{Tg}$

d.
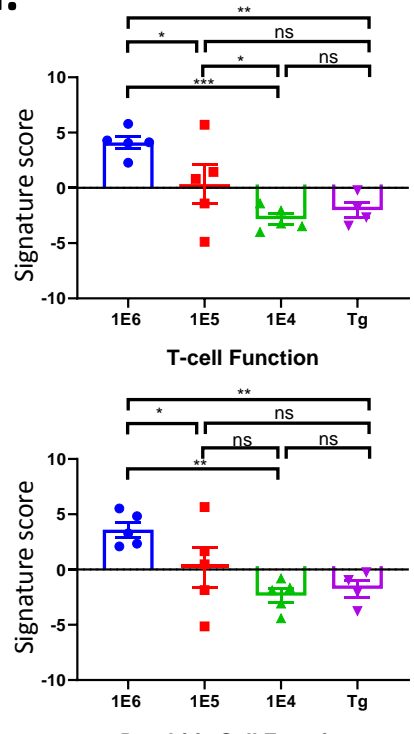

Dendritic Cell Functions
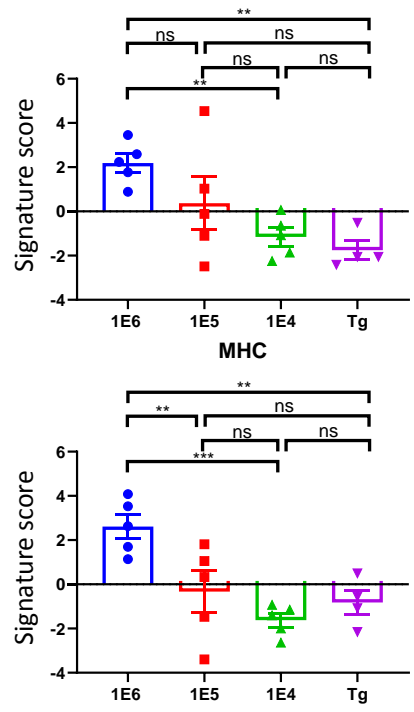

Macrophages

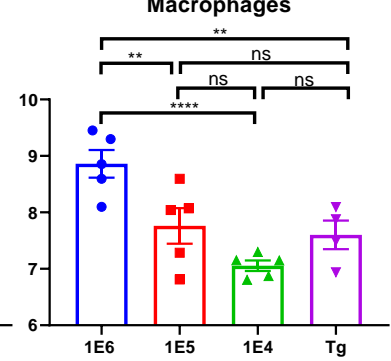

Adaptive
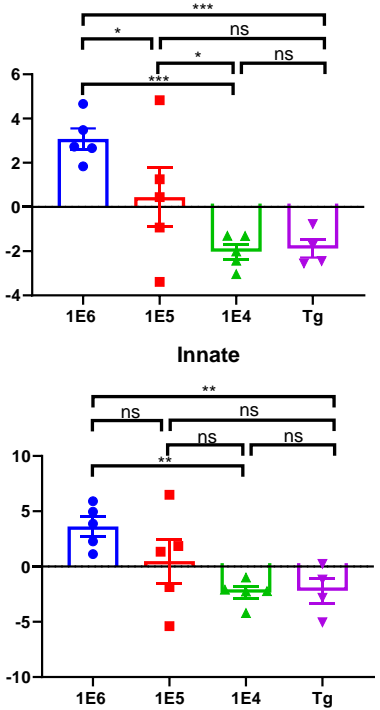

Macrophage Functions
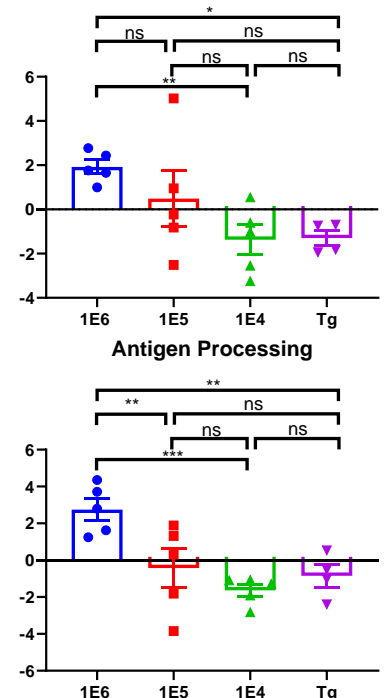

Ccr2

Ccl2
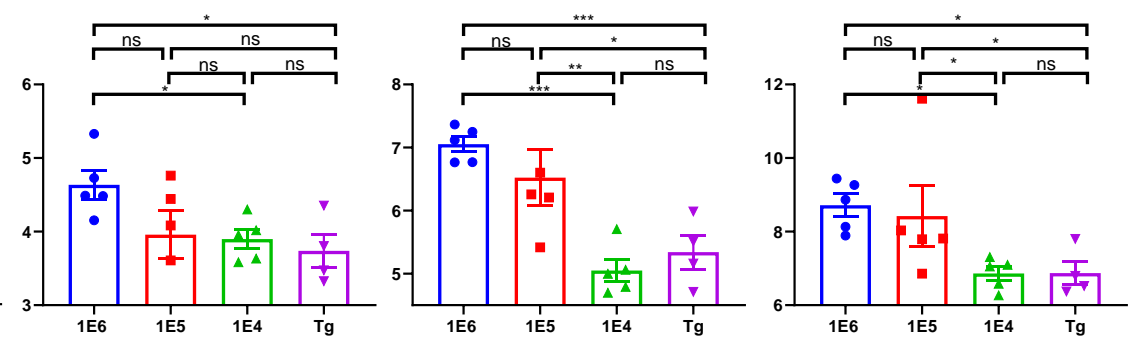
Castrillon et al. Figure 6. The MMTV-PyMT syngeneic models respond differently to immune checkpoint blockade.

a.
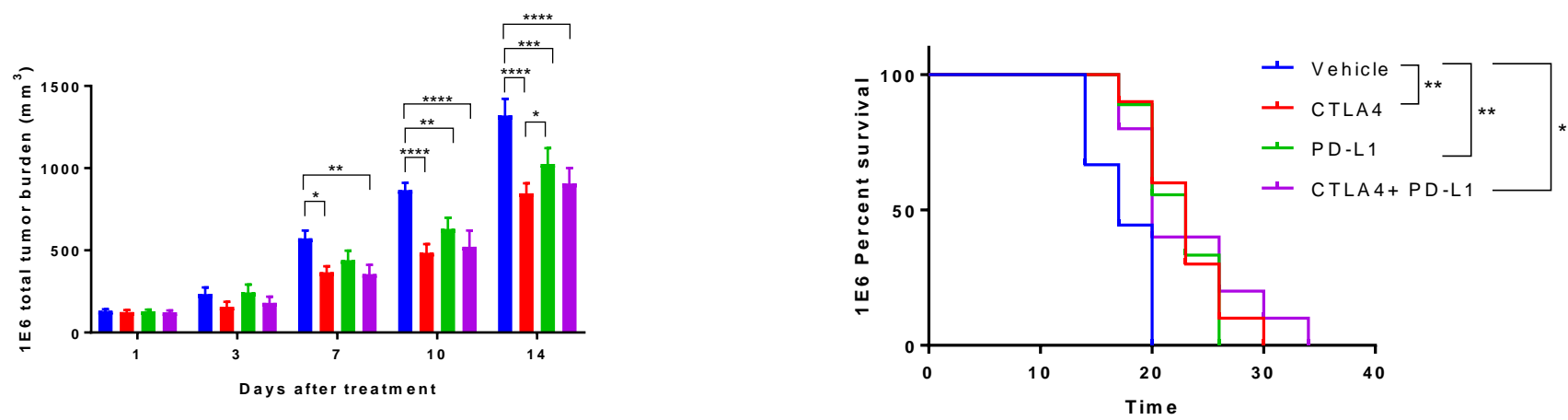

b.
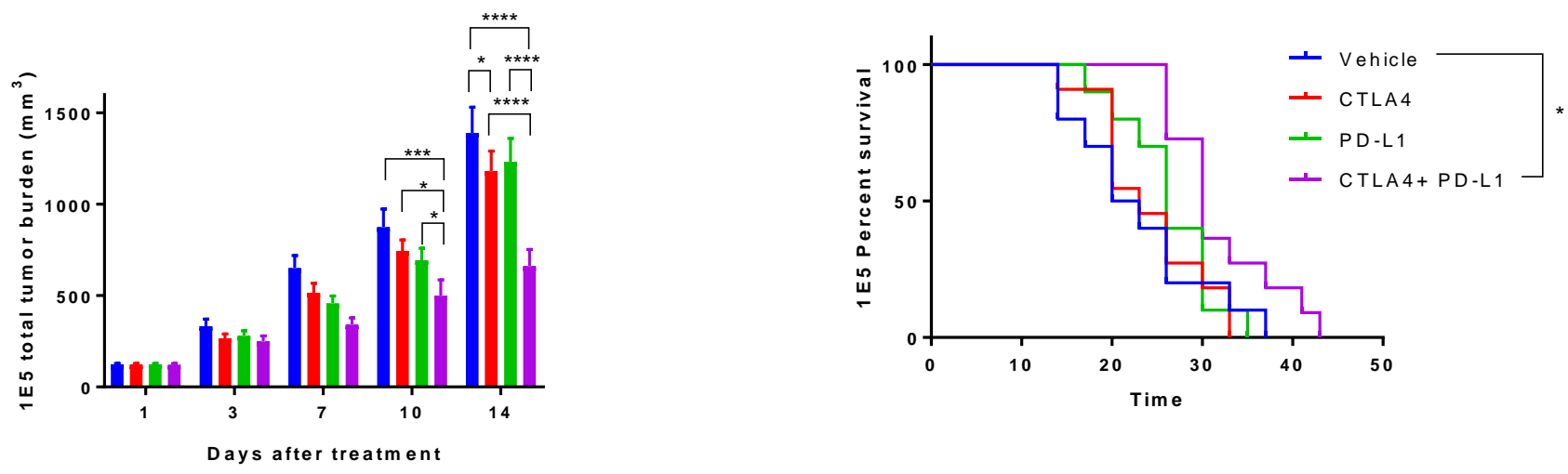

c.
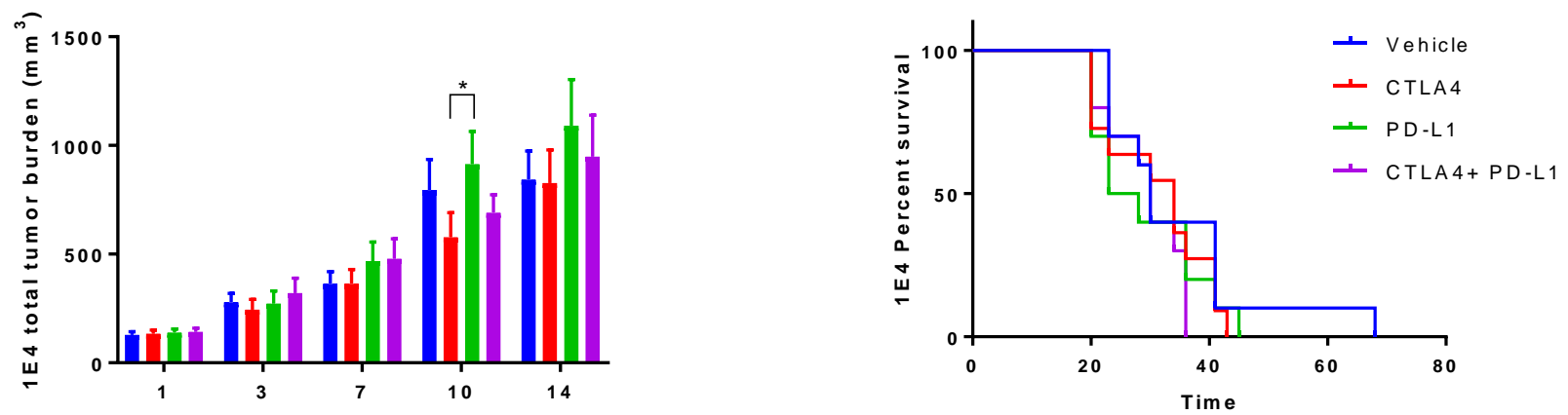

d.
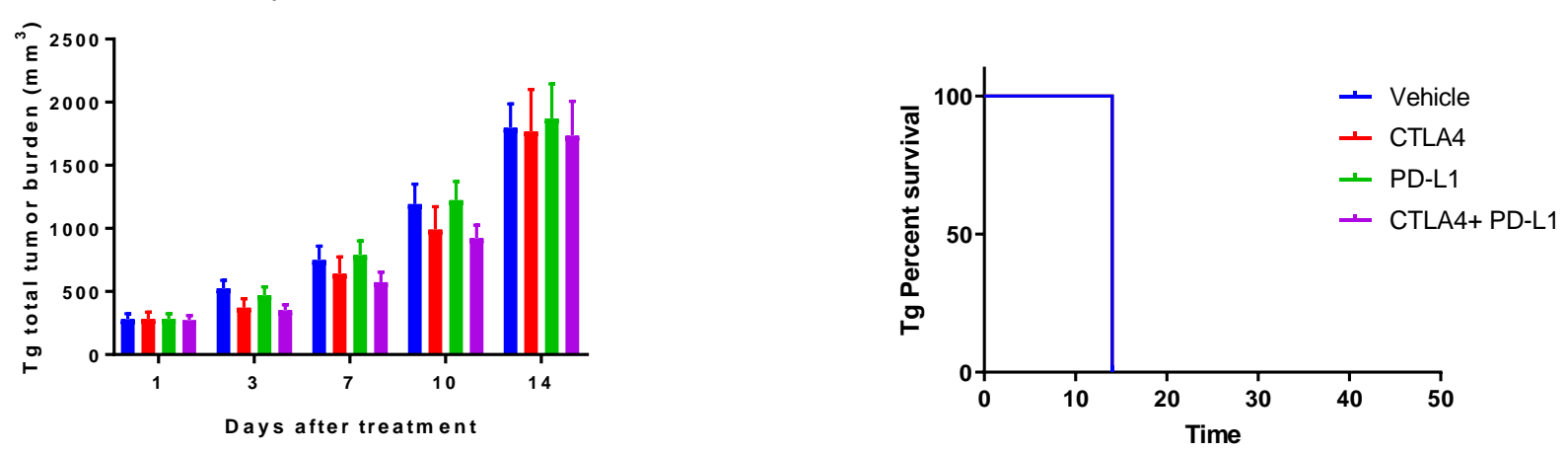
Castrillon et al. Figure 7. Summary Slide.

a. T cells

\begin{tabular}{|r|c|c|c|}
\hline & $1 \mathrm{E} 6$ & $1 \mathrm{E} 5$ & $1 \mathrm{E} 4$ \\
\hline Tumor growth & +++ & ++ & + \\
\hline CD45+/Viable & +++ & ++ & + \\
\hline CD3+/viable & +++ & ++ & + \\
\hline CD8+/viable & +++ & ++ & + \\
\hline CD4+/viable & +++ & ++ & + \\
\hline CD11b+/viable & + & + & - \\
\hline Ratio: CD3+/CD11b of viable & + & + & - \\
\hline CD3+/CD45+ & ++ & ++ & ++ \\
\hline CD8+/CD45+ & + & + & + \\
\hline CD4+/CD45+ & ++ & ++ & + \\
\hline Ratio: CD4+ of CD3+/CD8+ of CD3+ & + & - & - \\
\hline GrB+/CD8+CD45+ & - & + & + \\
\hline RoxP3+/CD4+CD45+ & + & + & + \\
\hline PD-1+/CD3+ & + & + & + \\
\hline
\end{tabular}

\section{Myeloid Cells}

Myeloid Cells
\begin{tabular}{|r|c|c|c|}
\hline CD11b+/CD45+ & $1 \mathrm{E} 6$ & $1 \mathrm{E} 5$ & $1 \mathrm{E} 4$ \\
\hline F480+/CD45+ & + & + & + \\
\hline Gr1+CD11b+/CD45+ & - & - & - \\
\hline CD11C+/CD45+ & - & - & - \\
\hline Gr1+/CD11b+ & + & + & - \\
\hline PD-L1+F480+/CD11b+ & ++ & ++ & + \\
\hline PD-L1+/CD11b+ & + & + & + \\
\hline PD-L1-F480+/CD11b+ & + & + & + \\
\hline PD-L1+CD45-/Viable & - & - & - \\
\hline CD40+/CD11b+ & + & + & + \\
\hline CD80+/CD11b+ & + & + & - \\
\hline CD86+/CD11b+ & + & - & - \\
\hline CD206+/CD11b+ & - & - & + \\
\hline CD40+/F480b+ & + & + & + \\
\hline CD80+/F480+ & + & + & + \\
\hline CD86+/F480+ & - & - & - \\
\hline CD206+/F480+ & - & + & - \\
\hline Ratio: M1/M2 macrophages & + & + & + \\
\hline Antigen processing/MHC & ++ & - & - \\
\hline CD11b+/CD3+ of CD45 & + & - & - \\
\hline
\end{tabular}

\section{ICB Efficacy}

\begin{tabular}{|r|c|c|c|c|}
\hline & $1 \mathrm{E} 6$ & $1 \mathrm{E} 5$ & $1 \mathrm{E} 4$ & $\mathrm{Tg}$ \\
\hline CTLA-4 monotherapy & + & + & - & - \\
\hline PD-L1 monotherapy & + & - & - & - \\
\hline PD-L1 + CTLA-4 combination therapy & ++ & ++ & - & - \\
\hline
\end{tabular}

b.

1E6

1E5
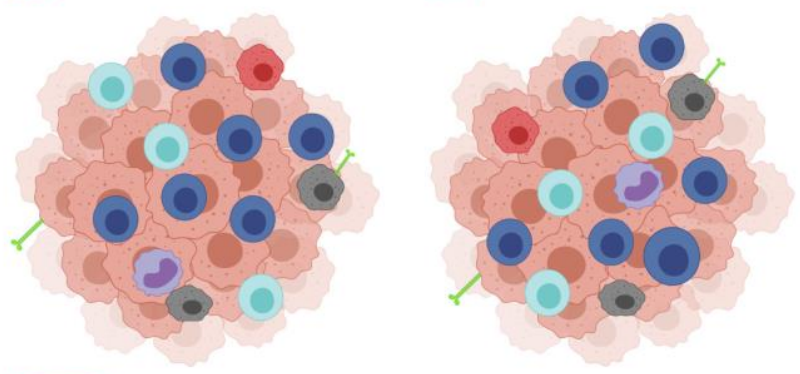

+ Significant compared to MMTV-PyMT

- Not significant compared to MMTV-PyMT

Increase compared to MMTV-PyMT

Decrease compared to MMTV-PyMT

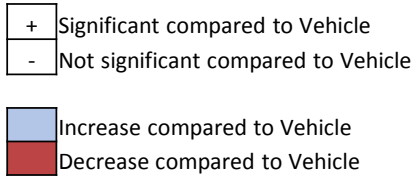

1E4

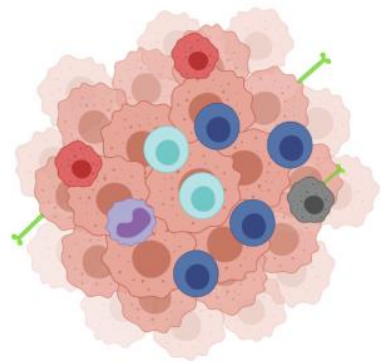

$\mathrm{Tg}$

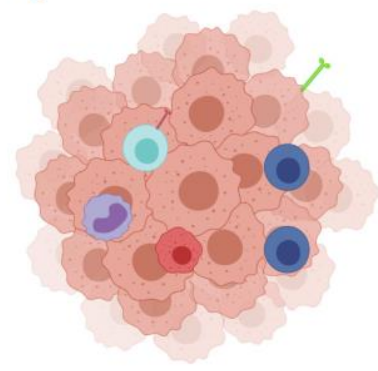


Castrillon et. al. Supplemental Figure 1. Immunophenotyping of cells used for generation of syngeneic murine models.

a.
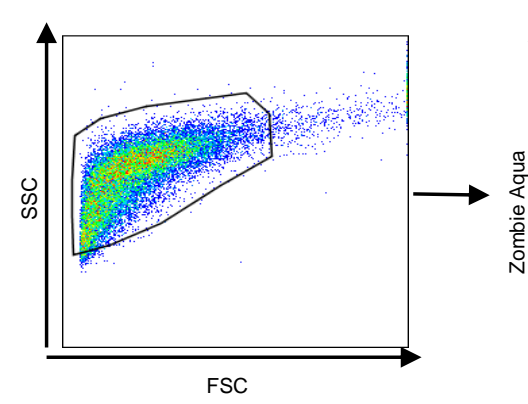

b.

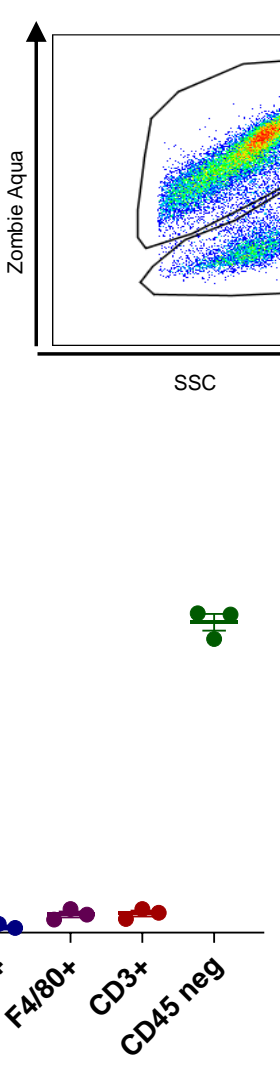

C

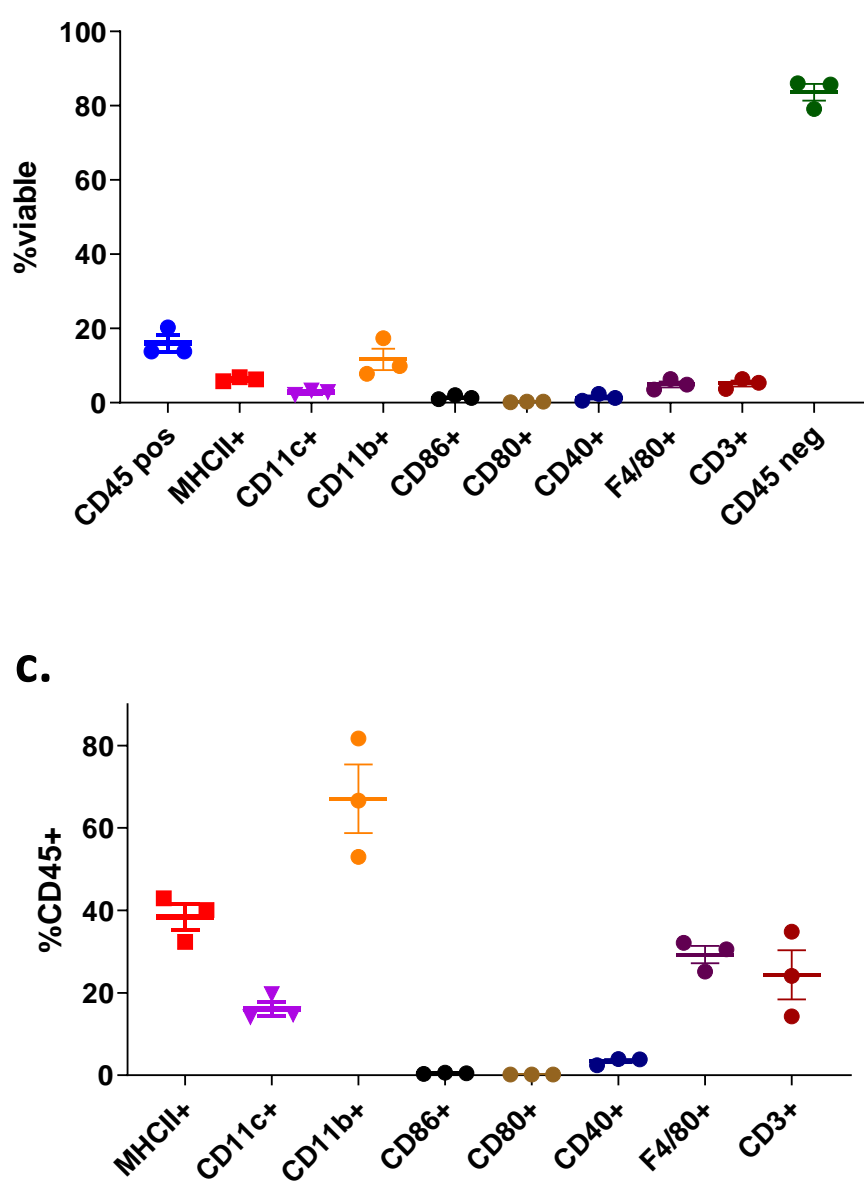

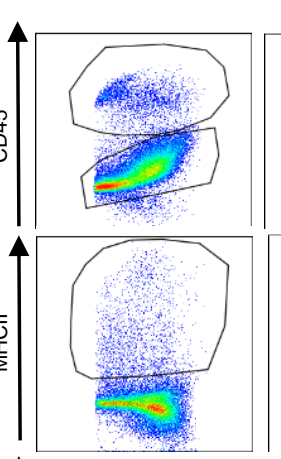

Negative Control
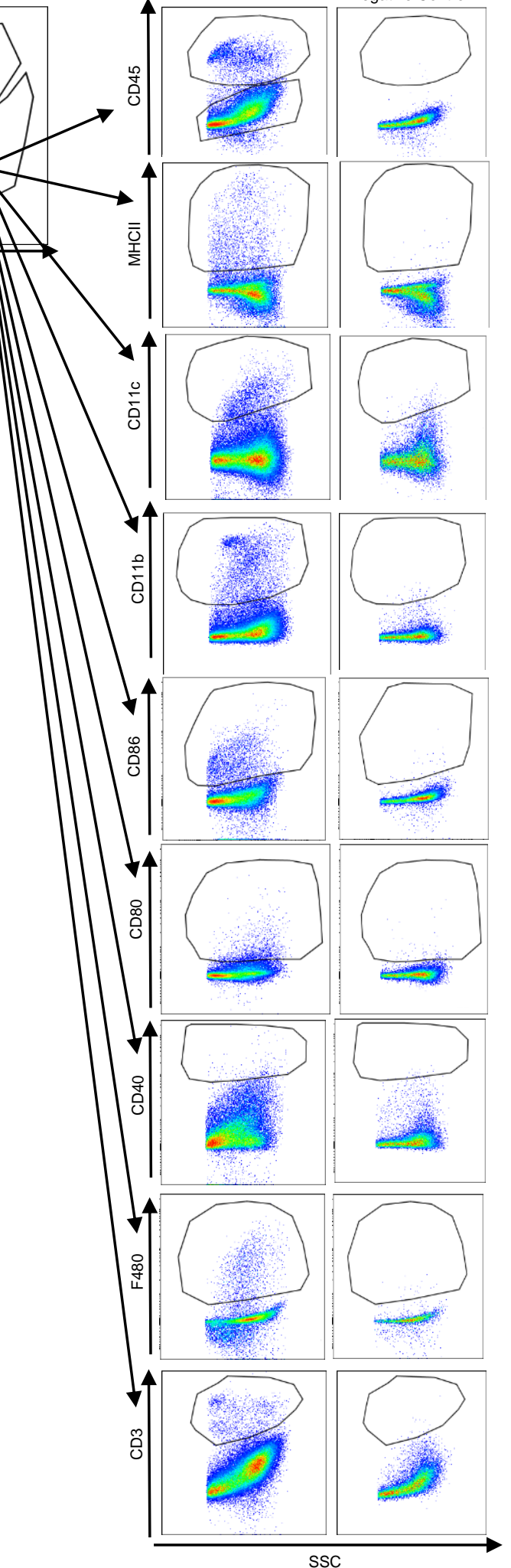


\section{Castrillon et al. Supplemental Figure 2. Example of FACs gating strategy.}

a.
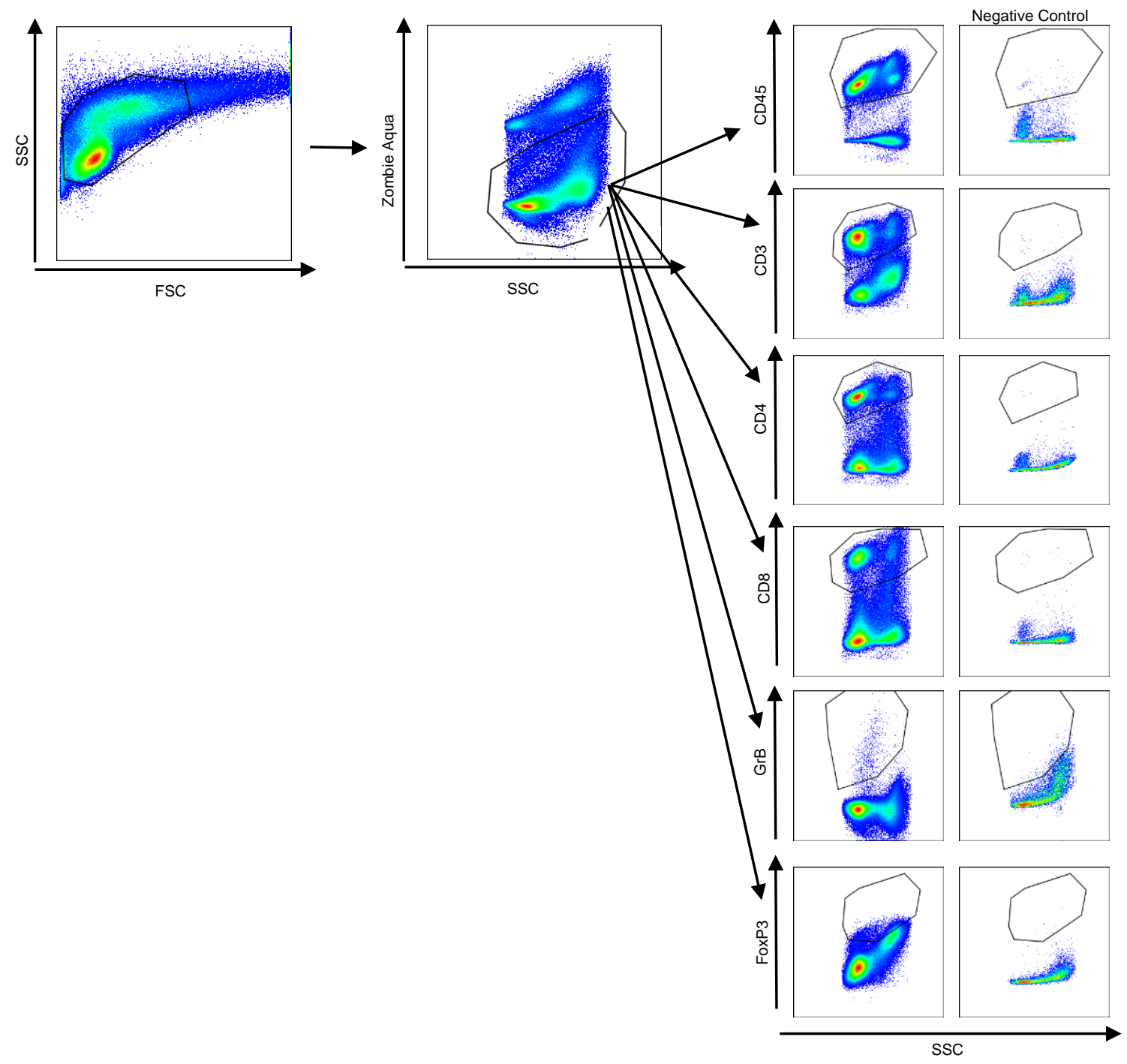
Castrillon et. al. Supplemental Figure 4. QC of mRNA expression analysis and cell type scores.

a.

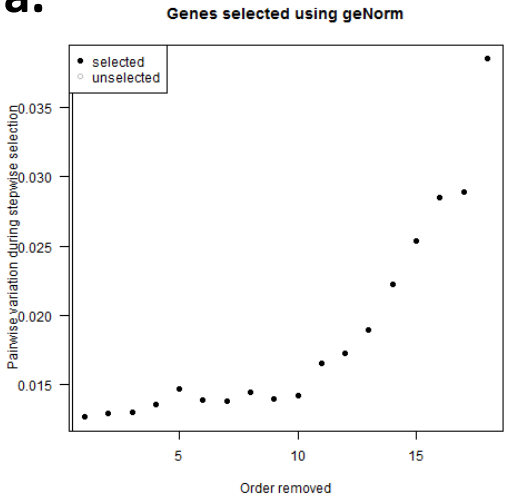

b.

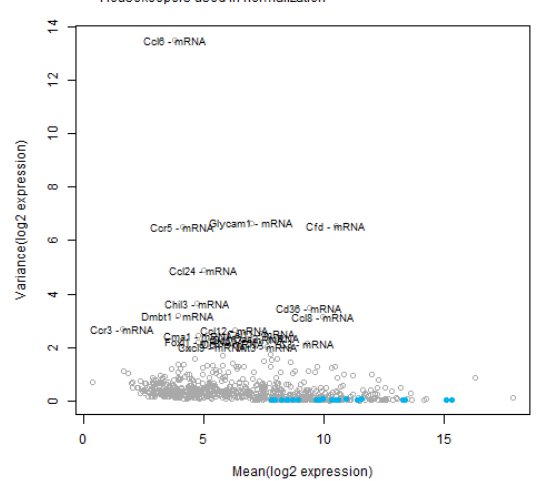

c.

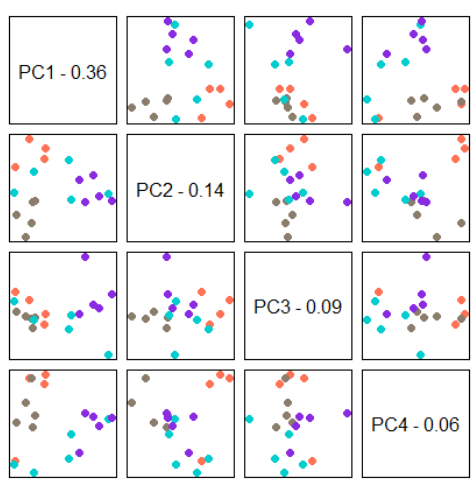

$1 \mathrm{E} 6$

1E4

$\mathrm{Tg}$ d.

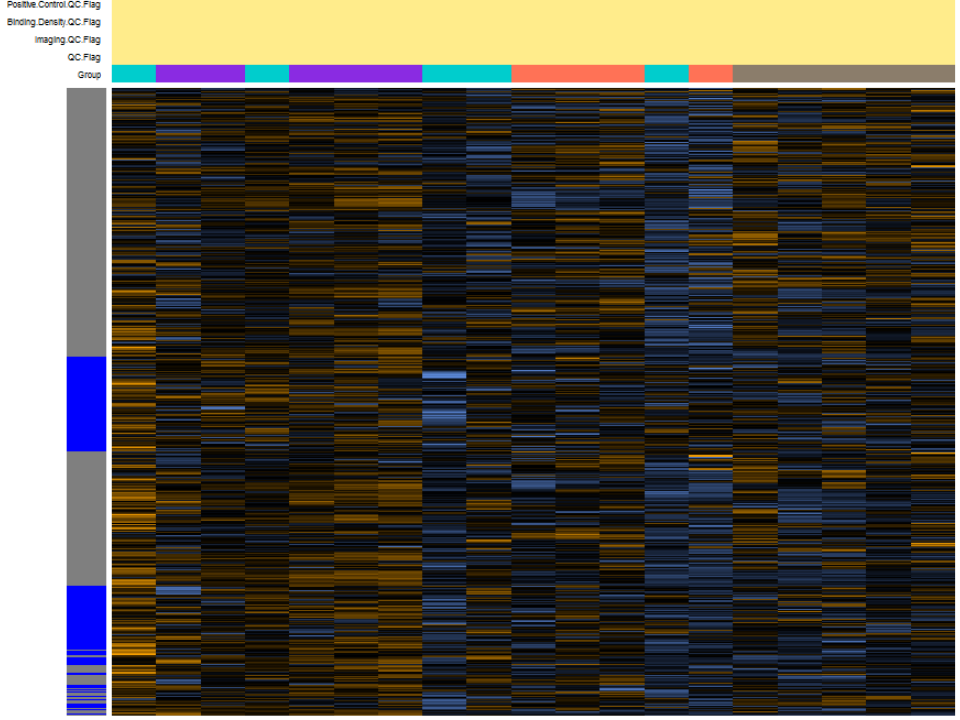

Probe Annotations

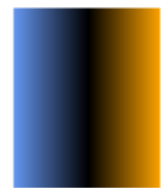

QC Flags

$\square$ True

flag/prune

-3-2-10123

z-scores
$1 \mathrm{E} 6$

$1 \mathrm{E5}$

$1 \mathrm{E} 4$

$\mathrm{Tg}$ e.

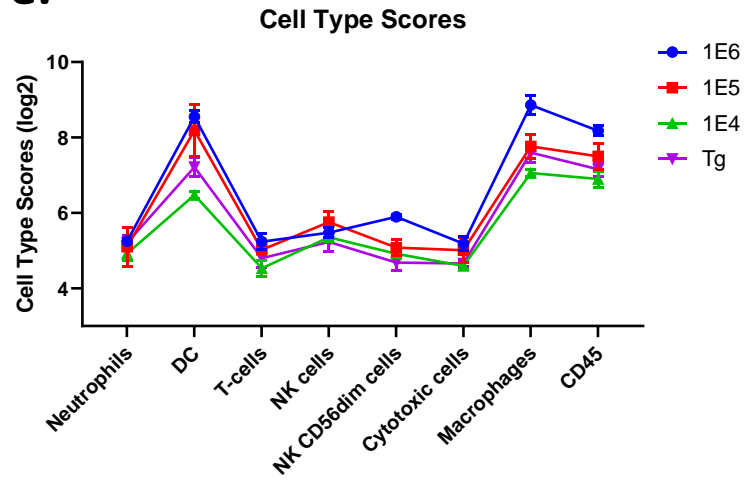

f.
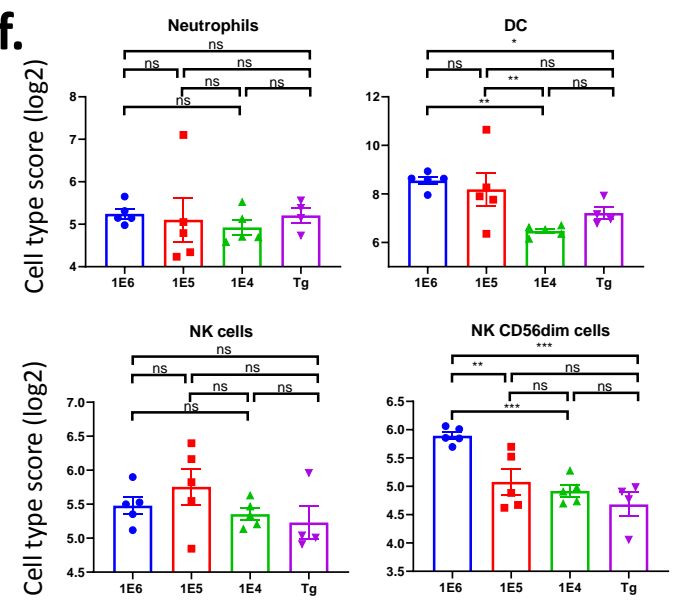
Castrillon et. al. Supplemental Figure 6. Pathway analysis by NanoString.

a.
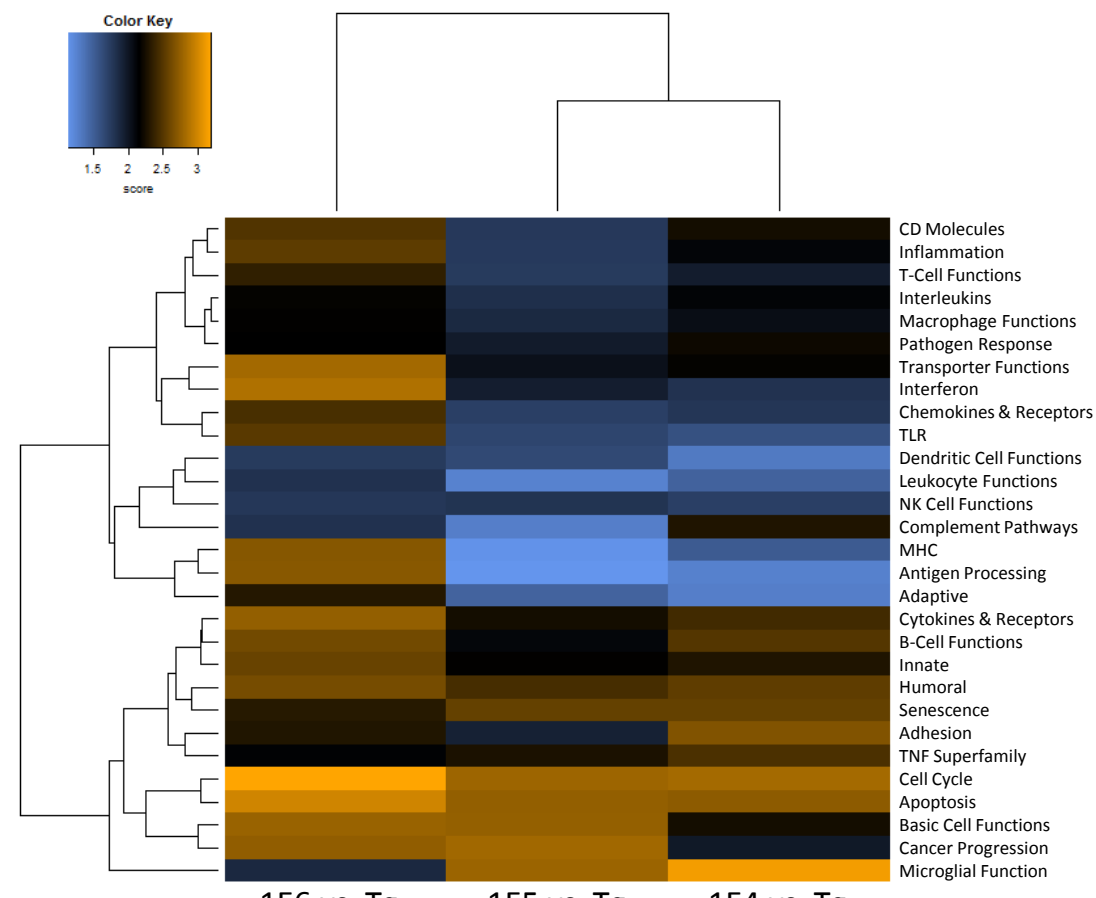

$1 \mathrm{E} 6$ vs. $\mathrm{Tg} \quad 1 \mathrm{E} 5$ vs. $\mathrm{Tg} \quad 1 \mathrm{E} 4$ vs. $\mathrm{Tg}$ b.

$1 \mathrm{E} 6 \mathrm{vs} \mathrm{Tg}$

b.

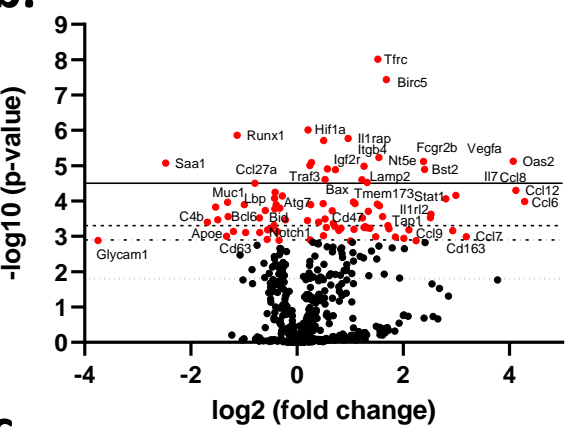

c.

$1 \mathrm{E5}$ vs Tg

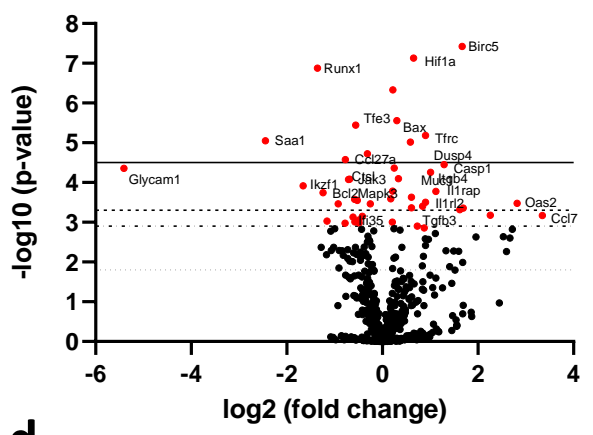

d.

$1 \mathrm{E} 4$ vs Tg

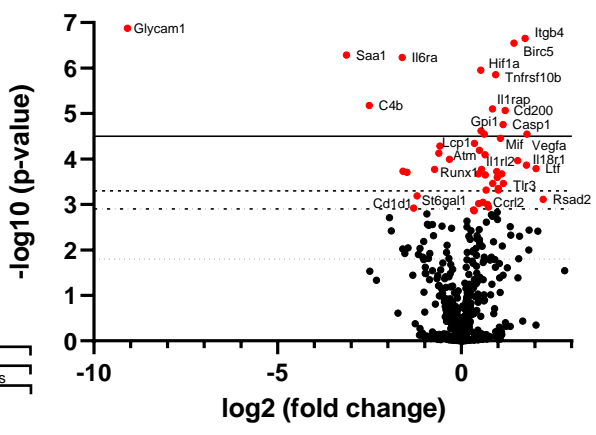

e.
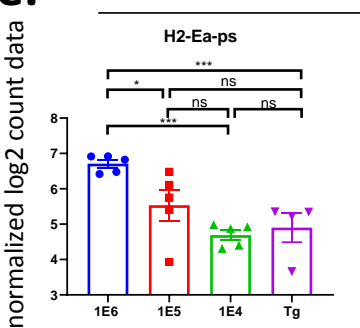

Antigen presentation pathway
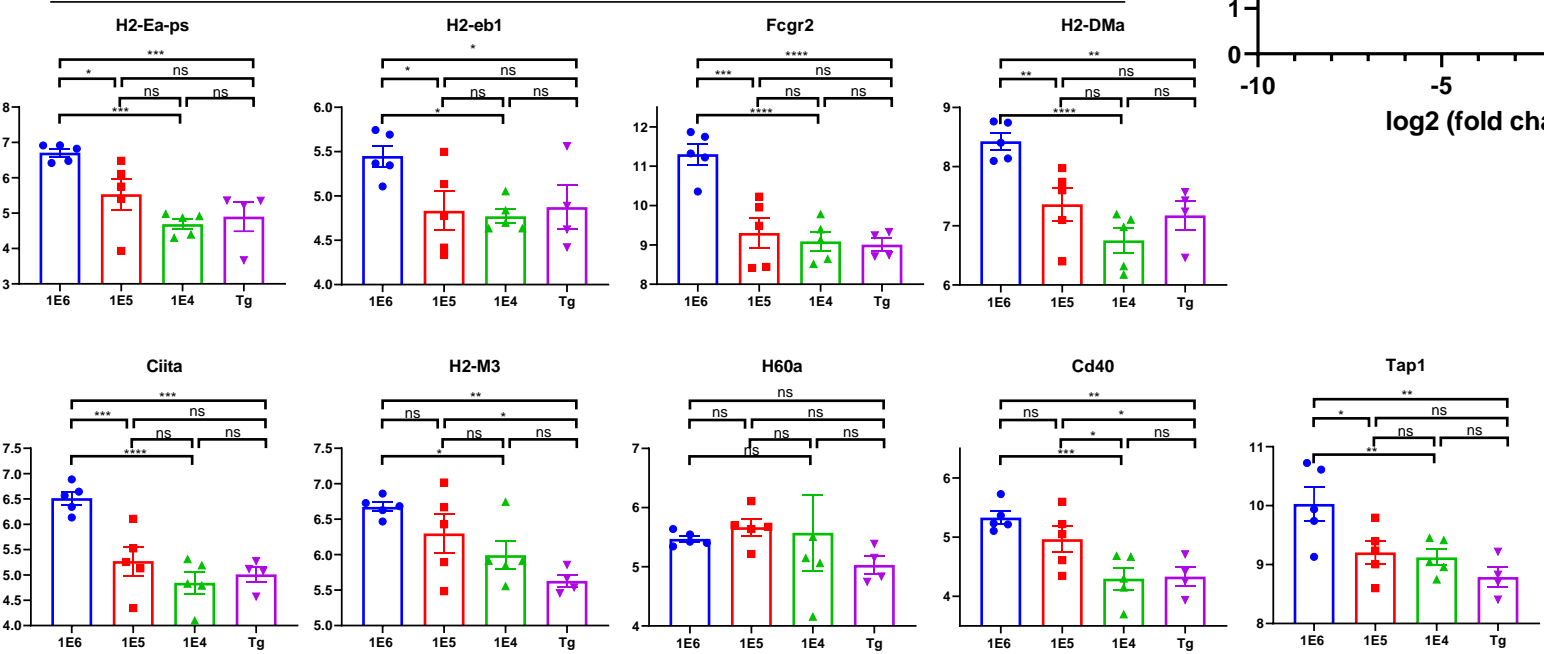

f.

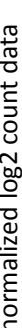
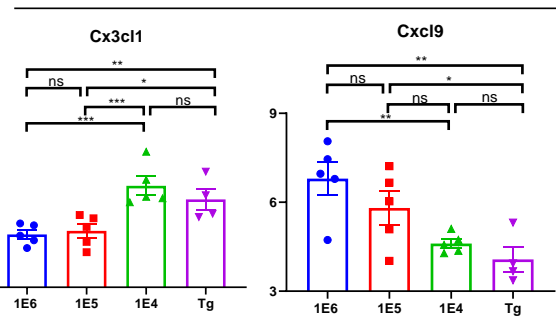

Immune signaling
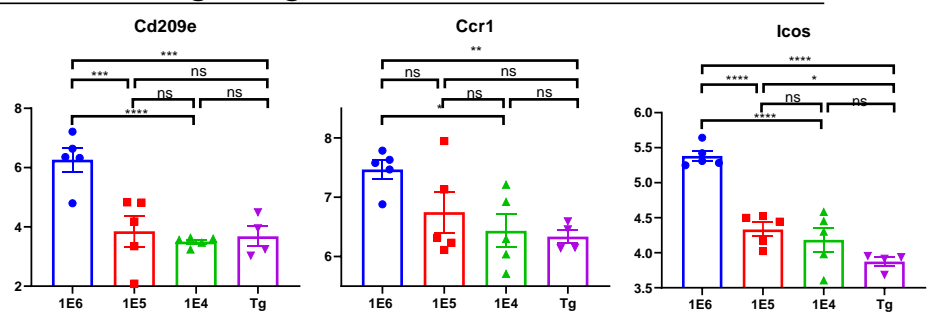

- $1 \mathrm{E} 6$ 

show significant differences in tumor fold change.

a.

b.
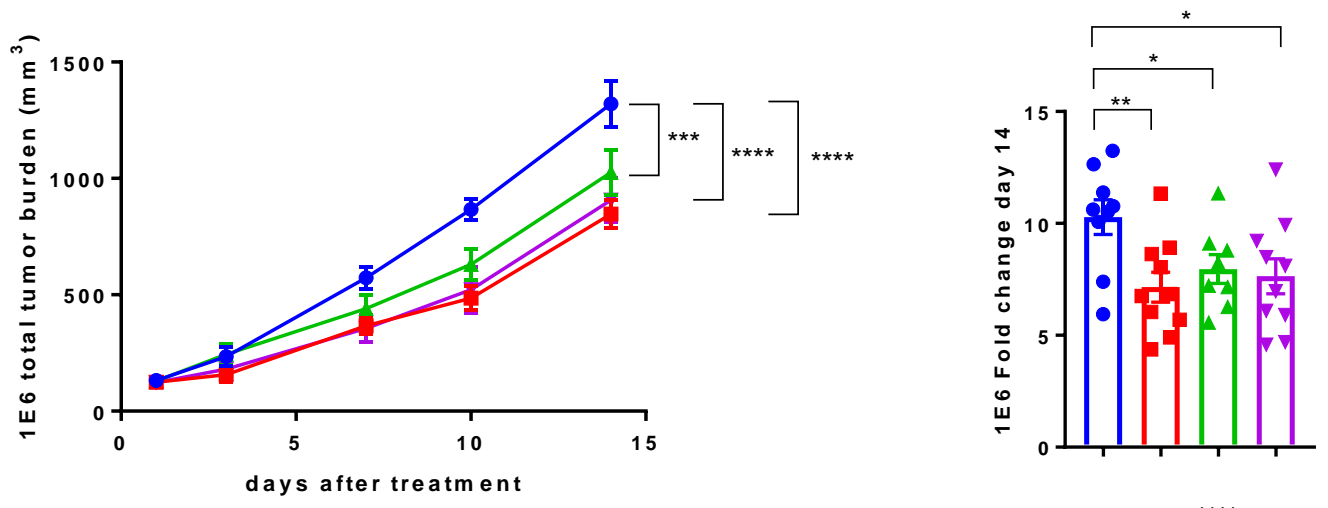

c.
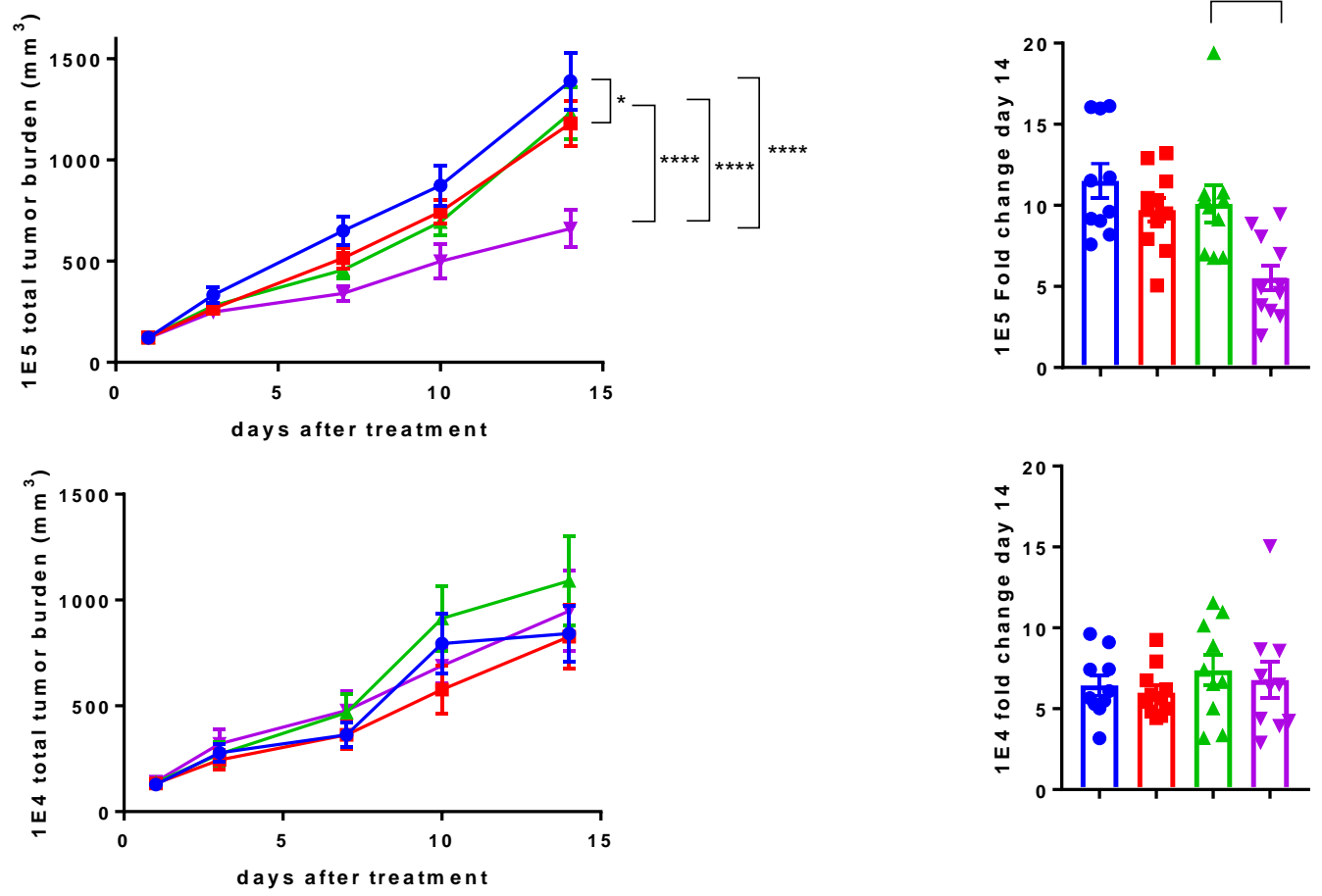

d.
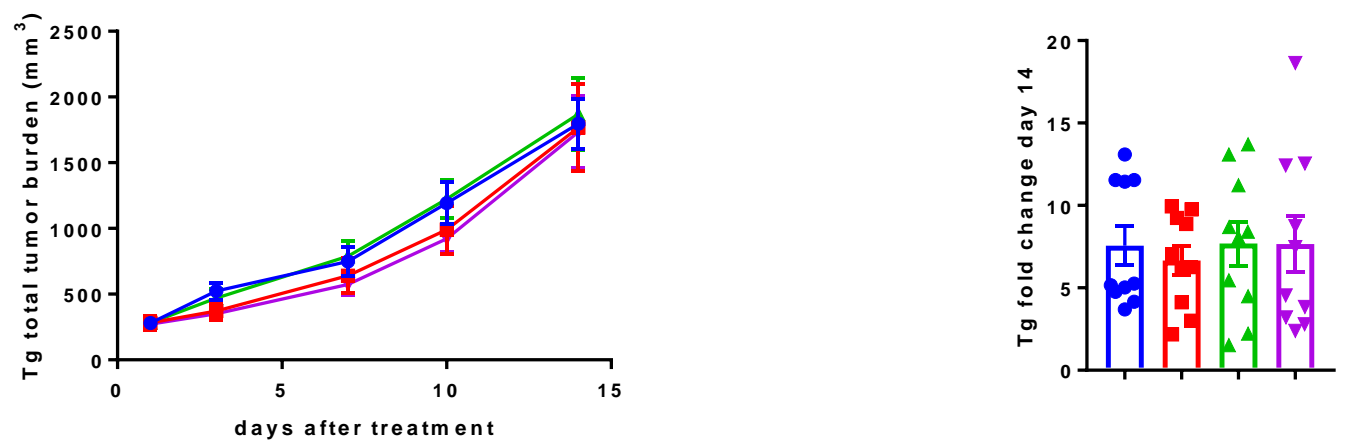
Castrillon et. al. Supplemental Figure 8. Comparison of predicted responders vs. non-responders by NanoString analysis.

a.

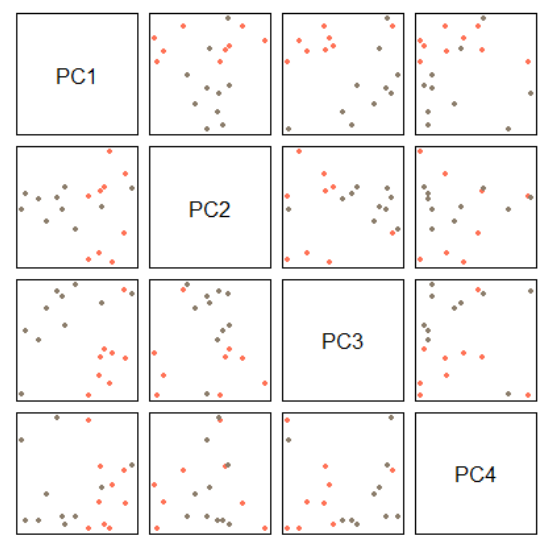

Predicted responder

Predicted non-responder b.

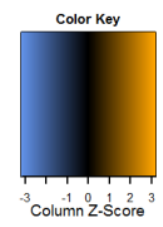

Cytotoxic

c.
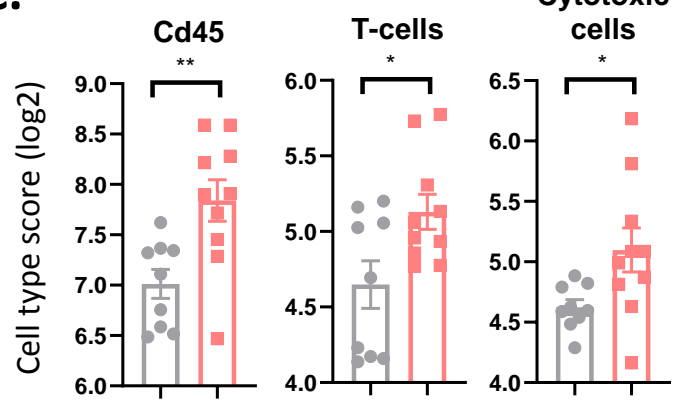

NK
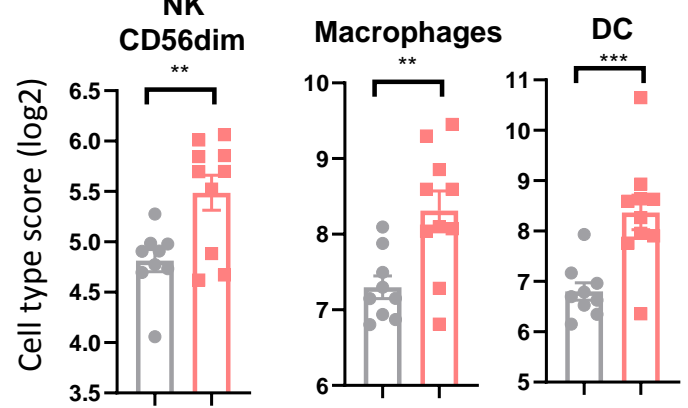

e.

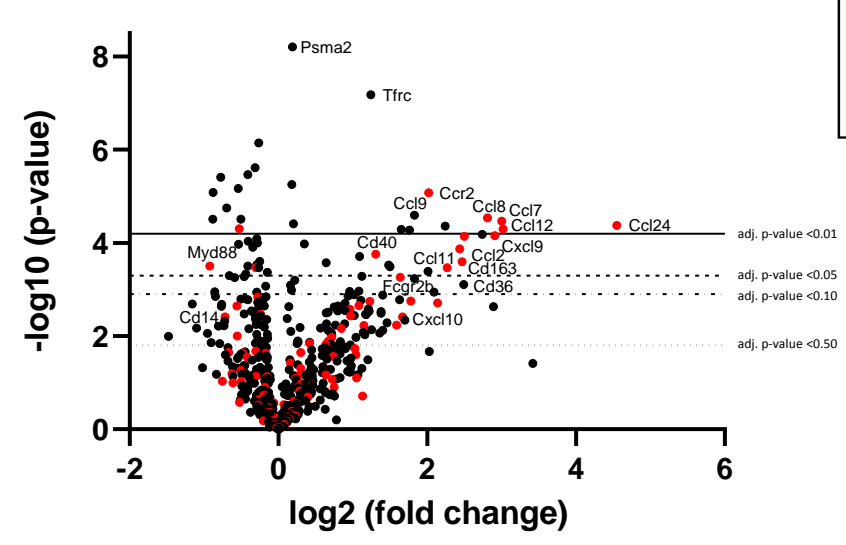

mRNA Inflammation score probe set

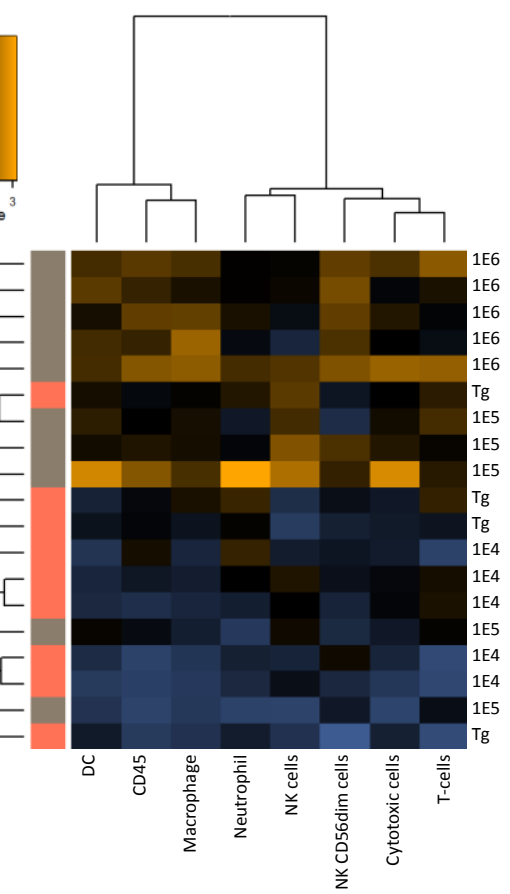

d.

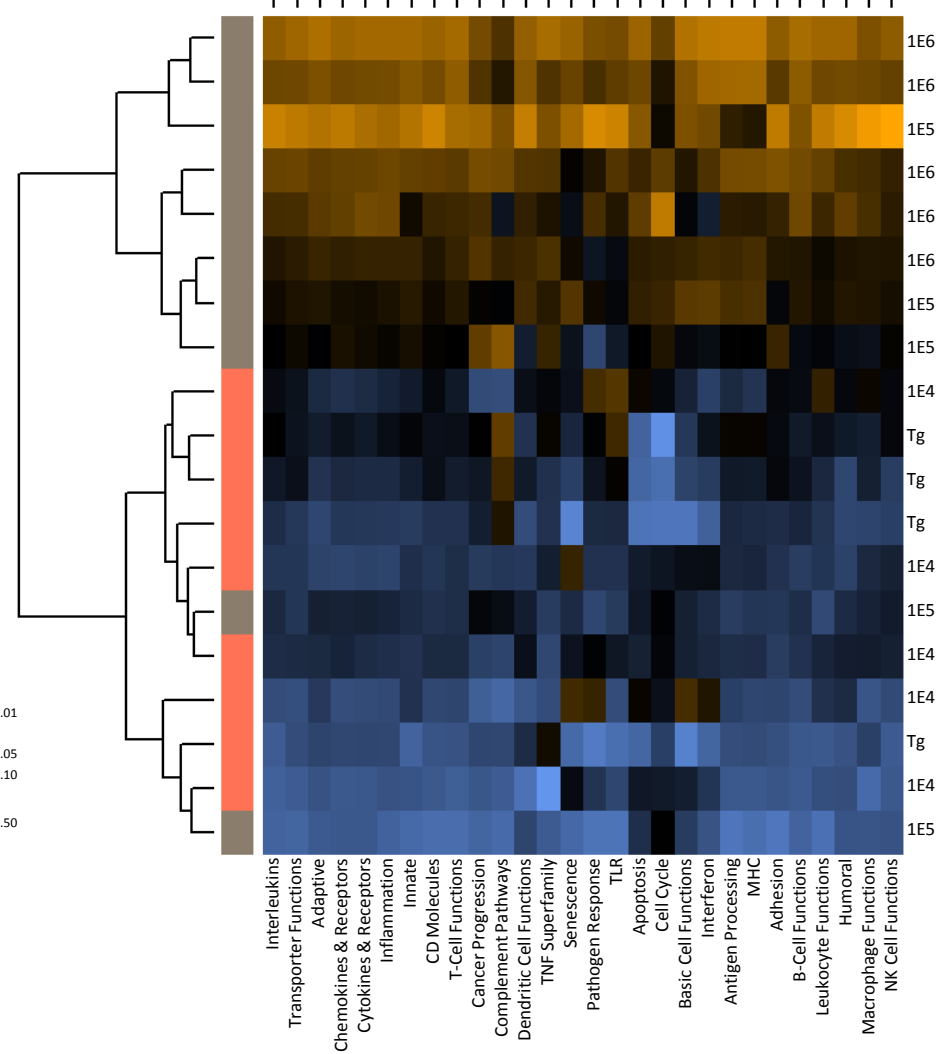


Table 1. List of Top 20 differential expression of immune transcripts in $1 \mathrm{E} 6$ versus the autochthonous model.

\begin{tabular}{|c|c|c|c|c|}
\hline Transcript & Gene.sets & probe.ID & Log2 fold change & P-value \\
\hline Tfrc-mRNA & CD molecules, Transporter Functions & NM_011638.3:1930 & 1.52 & $9.64 \mathrm{E}-09$ \\
\hline Birc5-mRNA & Apoptosis, Cell Cycle, Cytokines \& Receptors & NM_009689.2:237 & 1.68 & $3.66 \mathrm{E}-08$ \\
\hline Psma2-mRNA & Cancer Progression & NM_008944.2:136 & 0.206 & $9.74 \mathrm{E}-07$ \\
\hline Runx1-mRNA & & NM_001111021.1:3055 & -1.13 & $1.38 \mathrm{E}-06$ \\
\hline II1rap-mRNA & Cytokines \& Receptors, Inflammation, Innate, Interleukins & NM_008364.2:2415 & 0.961 & $1.70 \mathrm{E}-06$ \\
\hline Hif1a-mRNA & Apoptosis, Cancer Progression & NM_010431.2:1294 & 0.503 & $1.94 \mathrm{E}-06$ \\
\hline Nt5e-mRNA & B-Cell Functions, CD molecules, Inflammation & NM_011851.3:1600 & 1.54 & $5.90 \mathrm{E}-06$ \\
\hline Oas2-mRNA & Basic Cell Functions & NM_145227.3:414 & 4.07 & $7.48 \mathrm{E}-06$ \\
\hline Fcgr2b-mRNA & Antigen Processing, B-Cell Functions, CD molecules, Inflammation, Interleukins, MHC, Transporter Functions & NM_001077189.1:1225 & 2.38 & $7.55 \mathrm{E}-06$ \\
\hline Ltbr-mRNA & Apoptosis, TNF Superfamily & NM_010736.3:1962 & 0.274 & $8.11 \mathrm{E}-06$ \\
\hline Saa1-mRNA & Adhesion, Cytokines \& Receptors, Innate, Macrophage Functions & NM_009117.3:351 & -2.47 & $8.41 \mathrm{E}-06$ \\
\hline Smn1-mRNA & Cancer Progression & NM_011420.2:390 & 0.24 & $9.88 \mathrm{E}-06$ \\
\hline Itgb4-mRNA & Adhesion, CD molecules & NM_001005608.2:3355 & 1.26 & $1.04 \mathrm{E}-05$ \\
\hline Bax-mRNA & Apoptosis, Transporter Functions & NM_007527.3:735 & 0.573 & $1.24 \mathrm{E}-05$ \\
\hline Bst2-mRNA & CD molecules, Humoral, Innate & NM_198095.2:468 & 2.4 & $1.28 \mathrm{E}-05$ \\
\hline Igf2r-mRNA & Apoptosis, CD molecules, Transporter Functions & NM_010515.1:2585 & 0.723 & $1.30 \mathrm{E}-05$ \\
\hline Traf3-mRNA & Apoptosis, Cytokines \& Receptors, Innate, TLR & NM_001048206.1:6385 & 0.526 & $2.45 \mathrm{E}-05$ \\
\hline Ikzf2-mRNA & T-Cell Functions & NM_011770.4:7230 & 1.22 & $2.52 \mathrm{E}-05$ \\
\hline Ddx58-mRNA & Innate, Interferon & NM_172689.3:1751 & 1.32 & $3.00 \mathrm{E}-05$ \\
\hline Ccl27a-mRNA & Cytokines \& Receptors & NM_001048179.1:265 & -0.793 & $3.15 \mathrm{E}-05$ \\
\hline
\end{tabular}


Table 2. List of Top 20 differential expression of immune transcripts in 1E5 versus autochthonous model.

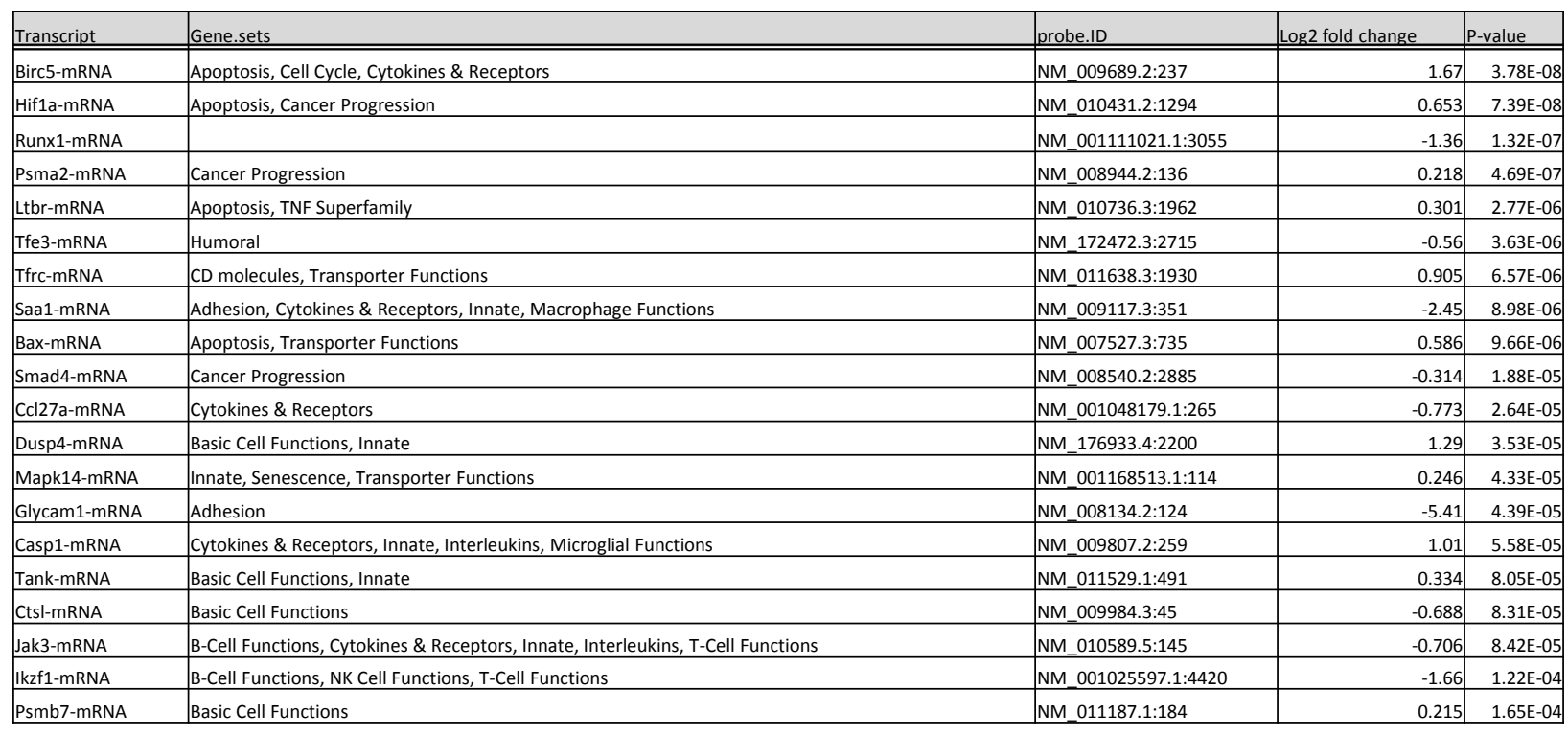


Table 3. List of Top 20 differential expression of immune transcripts in 1E4 versus autochthonous model.

\begin{tabular}{|c|c|c|c|c|}
\hline Transcript & Gene sets & probe.ID & Log 2 fold change & P-value \\
\hline Glycam1-mRNA & Adhesion & NM_008134.2:124 & -9.09 & $1.34 \mathrm{E}-07$ \\
\hline Itgb4-mRNA & Adhesion, CD molecules & NM_001005608.2:3355 & 1.73 & $2.23 \mathrm{E}-07$ \\
\hline Birc5-mRNA & Apoptosis, Cell Cycle, Cytokines \& Receptors & NM_009689.2:237 & 1.43 & $2.83 \mathrm{E}-07$ \\
\hline $116 \mathrm{ra}-\mathrm{mRNA}$ & CD molecules, Chemokines \& Receptors, Cytokines \& Receptors, Interleukins & NM_010559.2:2825 & -1.61 & $5.86 \mathrm{E}-07$ \\
\hline Hif1a-mRNA & Apoptosis, Cancer Progression & NM_010431.2:1294 & 0.527 & $1.12 \mathrm{E}-06$ \\
\hline Tnfrsf10b-mRNA & Apoptosis, CD molecules, TNF Superfamily & NM_020275.3:1625 & 0.934 & $1.40 \mathrm{E}-06$ \\
\hline Cd200-mRNA & CD molecules & NM_010818.3:686 & 1.19 & $8.63 \mathrm{E}-06$ \\
\hline Casp1-mRNA & Cytokines \& Receptors, Innate, Interleukins, Microglial Functions & NM_009807.2:259 & 1.13 & $1.76 \mathrm{E}-05$ \\
\hline Gpi1-mRNA & Apoptosis, Cytokines \& Receptors, Humoral & NM_008155.4:1540 & 0.536 & $2.43 \mathrm{E}-05$ \\
\hline Mif-mRNA & B-Cell Functions, Cytokines \& Receptors, Inflammation, Innate, Transporter Functions & NM_010798.2:373 & 0.623 & $2.84 \mathrm{E}-05$ \\
\hline Vegfa-mRNA & Apoptosis, Cytokines \& Receptors, Macrophage Functions, T-Cell Functions & NM_001025250.3:3015 & 1.78 & $2.85 \mathrm{E}-05$ \\
\hline Atm-mRNA & Apoptosis, B-Cell Functions, Cell Cycle, Senescence & NM_007499.2:5543 & -0.616 & $7.50 \mathrm{E}-05$ \\
\hline App-mRNA & Apoptosis, Cell Cycle, Innate, Transporter Functions & NM_007471.2:511 & 0.641 & $8.04 \mathrm{E}-05$ \\
\hline
\end{tabular}

